\title{
Stable heteroaromatic carbenes of the benzimidazole and 1,2,4- triazole series
}

\author{
Nikolai I. Korotkikh*a, Oles P. Shvaika ${ }^{a}$, Gennady F. Rayenko ${ }^{a}$, Artyom V. Kiselyov ${ }^{a}$, \\ Arthur V. Knishevitsky ${ }^{a}$, Alan H. Cowley ${ }^{b}$ Jamie N. Jones $^{b}$, and Charles L.B. Macdonald \\ ${ }^{a}$ The L.M.Litvinenko Institute of Physical Organic and Coal Chemistry, \\ Ukrainian Academy of Sciences, Donetsk, Ukraine \\ 70, R.Luxemburg, Donetsk, 83114, Ukraine \\ E-mail: korotkikh@infou.donetsk.ua \\ ${ }^{b}$ The University of Texas at Austin, Austin, USA \\ Department of Chemistry \& Biochemistry, the University of Texas at Austin, Austin, 78712,
} USA;

E-mail: Cowley@mail.utexas.edu

(received 01 Oct 04; accepted 14 Mar 05; published on the web 05 May 05)

\begin{abstract}
The review is focused on new data concerning the syntheses and selected properties of stable heteroaromatic monocarbenes of the benzimidazole and 1,2,4-triazole series, biscarbenes, anionocarbenes of the 1,2,4-triazole series, and cryptocarbenes. The syntheses of the precursors to these compounds are also described. Special attention is paid to $\mathrm{C}-\mathrm{H}$ insertion reactions, $\mathrm{X}$-ray structural data and spectral characteristics of the carbenes and their analogues.
\end{abstract}

Keywords: Heteroaromatic monocarbenes, biscarbenes, anionocarbenes, benzimidazol-2ylidene, 1,2,4-triazol-5-ylidenes, 2(5) $H$-azolines, cryptocarbenes

\section{Contents}

1 Introduction

2 Major approaches. Synthesis of precursors for monocarbenes of the 1,2,4-Triazole and benzimidazole series, biscarbenes and anionocarbenes of the 1,2,4-triazole series

2.1 Preparation of the 1,2,4-triazole series precursors

2.2 Preparation of the benzimidazole series precursors

2.3 Preparation of the 3,3'-bridged bistriazolium precursors

2.4 Preparation of the 4,4'-bridged bistriazolium precursors

2.5 Preparation of the 1,1'-bridged triazolium biscarbene precursors

2.6 A precursor with a functional $\mathrm{OH}$ group 
3 Heteroaromatic carbenes of the 1,2,4-triazole and benzimidazole series

3.1 Monocarbenes of the 1,2,4-triazole series

3.2 Monocarbene of the benzimidazole series

3.3 Heteroaromatic biscarbenes of the 1,2,4-triazole series

3.4 Stable chelate anionocarbene complexes

4 Structure of heteroaromatic carbenes, salts and 2(5) $H$-azolines

5 Properties of heteroaromatic carbenes

5.1 Spectral properties

5.2 Basicity

5.3 Insertion reactions into polar bonds

5.4 C-H Insertions

5.5 Reactions with chalcogens

5.6 Complex formation

5.7 Reactions of heteroaromatic carbenes with double bonds

5.8 Reactions of cryptocarbenes

6 Conclusions

7 References

\section{Introduction}

Following the discovery of rapid proton exchange in thiazolium salts by Breslow ${ }^{1}$, it became clear that cyclic ylidic compounds, otherwise known as neutral carbenes, can participate in chemical reactions. In numerous works Wanzlick and co-workers, along with many other authors, advanced the chemistry of stable nucleophilic carbenes of the thiazole, benzothiazole, imidazoline and imidazole series based on in situ experiments ${ }^{2}$. This area received a considerable impetus when Bertrand et $a l^{3,4}$ and Arduengo et al., ${ }^{5,6}$ isolated the first representatives of phosphanylsilylcarbenes and imidazol-2-ylidenes, respectively, thus demonstrating stability of singlet nucleophilic carbenes under normal experimental conditions. Subsequently, other new classes of stable carbenes have been reported, including acyclic diaminocarbenes ${ }^{7}, 1,2,4$-triazol5 -ylidenes ${ }^{8}$, and thiazol-2-ylidenes ${ }^{9}$. The majority of the carbenes obtained thus far are heterocyclic, and the most stable ones are heteroaromatic carbenes (HC). The stability of such heteroaromatic carbenes (HC's) is attributable to aromatic delocalization in a ring which involves participation of a vacant orbital of the carbene carbon atom.

Several methods for synthesis of stable carbenes have been developed. The method of Arduengo et $a l^{5,6}$, which is really a modification of Wanzlick's approach ${ }^{10}$, features deprotonation of azolium salts with sodium hydride and potassium tert-butoxide in tetrahydrofuran (THF). The method of Kuhn and $\mathrm{Kratz}^{11}$ involves the reductive desulfurization

of imidazolthiones by potassium in THF, and the method of Enders et al. ${ }^{8,12}$ consists of deprotonation of a 1,2,4-triphenyl-1,2,4-triazolium salt in alcohol to form 1,2,4-triphenyl-5- 
methoxy-5H-1,2,4-triazoline, which loses methanol upon heating to afford the corresponding triazolylidene. Alder and co-workers have subsequently modified the method of Arduengo et al. by using reagents such as lithium diisopropylamide or potassium bistrimethylsilylamide to effect deprotonation. These authors also discovered the complexation of alkali metal ions by carbenes 7,13. Herrmann and co-workers have reported a synthetic method based on the use of metal amides in liquid ammonia and its mixtures with THF. This method has proved successful for the synthesis of a variety of imidazol-2-ylidenes and biscarbenes ${ }^{14,15}$. Subsequently, other new types of stable carbenes and biscarbenes have been synthesized (for example pyridine-containing carbenes $^{16}$, biscarbenes ${ }^{17,18}$, and carbenes of the pyrimidine ${ }^{12}$ and perimidine ${ }^{19}$ series). The recent synthesis by Hahn et al. ${ }^{20}$ of stable, non-aromatic imidazolin-2-ylidenes, which are sterically protected on one side, and the modification of the carbene structure by the nucleophilic substitution of a phosphonio group in a phosphonioarylcarbene ${ }^{21}$ are especially interesting. In some cases, the pure crystalline carbenes were not isolated and the in situ prepared solutions of carbenes were used for the synthesis of metal complexes ${ }^{14,15}$. All the published methods for the preparation of stable nucleophilic carbenes require special experimental conditions to exclude moisture and oxygen by the use of dry boxes or Schlenk technique.

In terms of the chemical properties of stable carbenes, the reactivity toward electrophiles has received special emphasis, particularly transition metal compounds. The synthesis of carbene transition metal complexes has attracted considerable attention due to their high thermal stabilities and useful catalytic properties. Indeed, carbene-metal complexes are often more stable and possess superior catalytic behavior than the corresponding phosphane complexes. Moreover, a series of new reactions of stable carbenes with organic substrates has also been found. For more comprehensive discussions of the synthesis and properties of stable carbenes, the reader is referred to the reviews of Herrmann $e a_{l}{ }^{22}$ and Bertrand et $a l^{23}$. For an earlier summary of HC chemistry, see reference ${ }^{2}$.

It should be noted that so far, the discussion has focused exclusively on stable nucleophilic carbenes, especially heteroaromatic carbenes $(\mathrm{HC})$, all of which exist in a singlet ground state. However, it should be mentioned that the field of stable triplet carbenes is beginning to emerge. The recent work of Tomioka et al. is particularly significant ${ }^{24}$. Nevertheless, the stabilities of triplet carbenes are appreciably less than singlet carbenes. For example, the half-life time of the most stable triplet carbene, bis-(10-phenylanthryl)carbene, is only $19 \mathrm{~min}$ at room temperature in solution ${ }^{24}$ while many stable singlet carbenes can be stored for many months without noticeable decomposition. Finally, attention is drawn to the challenge of synthesizing heteroaromatic electrophilic carbenes. The search for such compounds and methods for their preparation is in progress. 


\section{Major approaches. Synthesis of precursors for monocarbenes of the 1,2,4- triazole and benzimidazole series and biscarbenes of the 1,2,4-triazole series}

Among the known classes of heteroaromatic carbenes, the benzimidazole derivatives have received the least attention thus far. In the case of 1,2,4-triazol-5-ylidenes, only one 1,3,4triphenyl derivative has been reported ${ }^{8}$. This 1,2,4-triazol-5-ylidene is an unusual system in that it features a smaller basicity and nucleophilicity than those of typical imidazol-2-ylidenes thereby exibiting enhanced stability. Prior to our study, only one crystalline triscarbene (a trisimidazol-2-ylidene) had been reported ${ }^{25}$ but no mention was made of its chemical properties or X-ray crystal structure. As mentioned above, several bisimidazol-2-ylidenes have been prepared; however, they are not sufficiently stable for prolonged storage ${ }^{16-18}$. It was therefore of interest to develop new methods for synthesis of such stable carbenes. In this work, we present new results in terms of the synthesis and properties of stable heteroaromatic monocarbenes and biscarbenes of the benzimidazole and 1,2,4-triazole series and their precursors. The methods for the precursor synthesis are also mentioned briefly.

Since the adamantyl group is one of the most bulky substituents and was employed for the synthesis of the first stable $\mathrm{HC}^{5}$, it was decided to employ this group in our work, along with other sterically demanding substituents such as tert-butyl and bulky aryls.

\subsection{Preparation of the 1,2,4-triazole series precursors}

The synthesis of a 1,2,4-triazole with aryl substituents in the 3 and 4 positions was carried out by the ring transformation of 2-phenyl-1,3,4-oxadiazoles $\mathbf{2 a , b}$ with anilines in the presence of trifluoroacetic acid (Scheme 1) ${ }^{26}$. The role of the latter is primarily as an acid catalyst; however, its presence also prevents the formation of colored impurities and the sublimation of some amines. Adamantylation of the 3,4-diaryl-substituted 1,2,4-triazoles 3a-d was carried out in acetic acid. The reaction proceeds smoothly and in high yields to afford 1-adamantyl-substituted 1,2,4-triazolium salts 1a-d ${ }^{27}$. Quaternization of the 2 position was not observed. Selective alkylation of triazoles in the 1 position was observed by treatment with tert-butyl iodide to form salt 1e. 

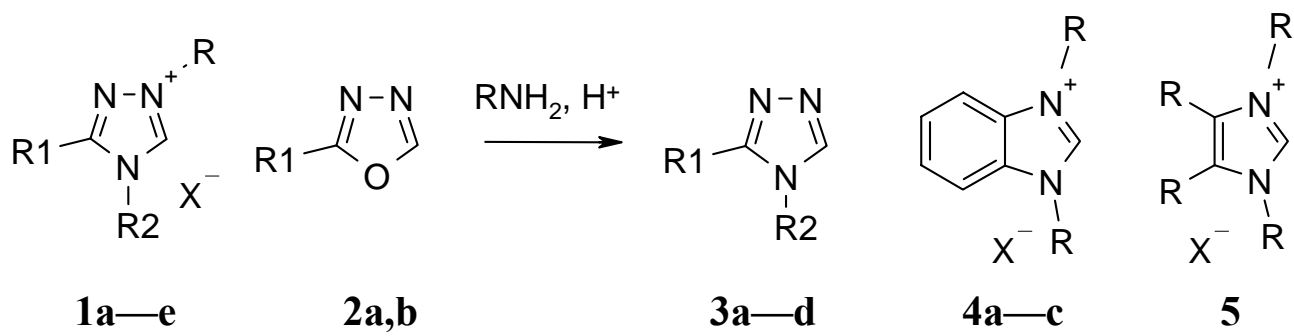

1a-d $\mathrm{R}=1$-Ad; 1 e $\mathrm{R}=t$-Bu; 1a,e,3a $\mathrm{R}_{1}=\mathrm{C}_{6} \mathrm{H}_{5}, \mathrm{R}_{2}=\mathrm{C}_{6} \mathrm{H}_{5} ; \mathbf{1 b}, \mathbf{3 b} \mathrm{R}_{1}=\mathrm{C}_{6} \mathrm{H}_{5}, \mathrm{R}_{2}=p$ - $\mathrm{BrC}_{6} \mathrm{H}_{4}$; 1c,3c $\mathrm{R}_{1}=\mathrm{R}_{2}=p-\mathrm{BrC}_{6} \mathrm{H}_{4} ; \mathbf{1 d}, \mathbf{3 d} \mathrm{R}_{1}=\mathrm{C}_{6} \mathrm{H}_{5}, \mathrm{R}_{2}=1-\mathrm{C}_{10} \mathrm{H}_{7} ; \mathbf{2 a} \mathrm{R}_{1}=\mathrm{C}_{6} \mathrm{H}_{5} ; \mathbf{2 b} \mathrm{R}_{1}=p-\mathrm{BrC}_{6} \mathrm{H}_{4}$; 4a $\mathrm{R}=\mathrm{CH}_{3} ; \mathbf{4 b ~} \mathrm{R}=\mathrm{CH}_{2} \mathrm{C}_{6} \mathrm{H}_{5} ; \mathbf{4 c} \mathrm{R}=1$-Ad; $5 \mathrm{R}=\mathrm{C}_{6} \mathrm{H}_{5}$.

\section{Scheme 1}

\subsection{Preparation of the benzimidazole series precursors}

One of the major aims in this area was the attachment of sterically bulky groups to the benzimidazole nucleus. As in the case of the 1,2,4-triazole series, 1-adamantyl was the substituent of choice. The introduction of the 1-adamantyl substituent into the benzimidazole system was achieved by direct adamantylation of benzimidazole using 1-bromoadamantane in the presence of sodium acetate in acetic acid ${ }^{28}$. However, in this case the reaction was incomplete and yields of only $33 \%$ of pure salt $4 \mathbf{c}$ were realized. In $o$-dichlorobenzene in the presence of potassium carbonate, a 54\% yield of 1-(1-adamantyl)benzimidazole can be achieved and this compound can be further quaternized by treatment with 1-bromoadamantane in $O$ dichlorobenzene to afford a high yield of salt $4 \mathbf{c}(90 \%)$. As is well known, in the presence of stronger bases benzimidazolium salts generate carbenes which undergo dimerization ${ }^{29}$. Finally, the most improved method for the synthesis of diadamantyl salt $\mathbf{4 c}$ comprises a one pot quaternization of benzimidazole by 1-bromoadamantane in $o$-dichlorobenzene in the presence of calcium hydride (the salt yield is $72 \%$ ). In this case the initial monoadamantylation proceeds effectively quantitatively and this in turn facilitates the subsequent quaternization. The salts with simple alkyl and aralkyl substituents $\mathbf{4 a , b}$ are easily accessible and can be obtained by the usual methods for azole alkylation in the presence of sodium acetate. Salt $\mathbf{5}$ was obtained by oxidation of 1,3,4,5-tetraphenylimidazol-2-thione with nitric acid according to the method described in reference ${ }^{30}$.

As conjugated bistriazolium salts were $3,3^{\prime}$ and 4,4'-bridged systems tested.

\subsection{Preparation of the 3,3'-bridged bistriazolium precursors}

For this goal a new method was developed that consists of ring transformations of the bridged 3,3'-arylene-bis-1,3,4-oxadiazoles $6 \mathrm{a}, \mathrm{b}$ by treatment with aromatic amines ${ }^{26,31}$ (Scheme 2). Similar ring transformations of 1,3,4-oxadiazoles under the action of amines and hydrazines have been described earlier ${ }^{32,33}$; however, these reactions only featured mononuclear 3,5-disubstituted systems. In the case of 5,5'-unsubstituted 3,3'-bridged bis-1,2,4-triazoles 7a,b, this represents the 
first time that this method has been employed. The reaction proceeds upon heating the mixture of bisoxadiazoles $6 \mathrm{a}$, b with amines at $200{ }^{\circ} \mathrm{C}$; however, abundant formation of colored impurities was observed. Furthermore, it was found that some amines undergo sublimation at these elevated temperatures. Under the conditions of trifluoroacetic acid catalysis in $o$-dichlorobenzene, the reaction is much cleaner and not accompanied by dye formation. Moreover, bistriazoles 7a,b are obtained in good yields (95\% and 46\%, respectively).

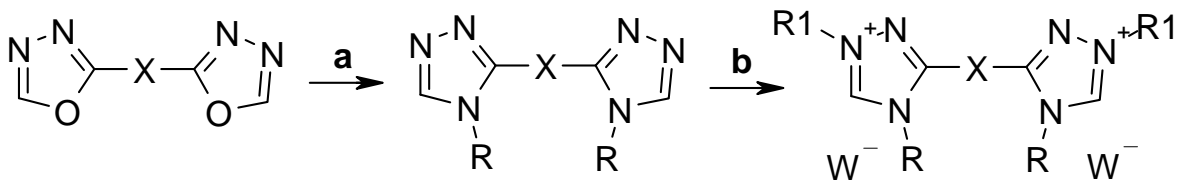

$6 a, b$

$7 \mathrm{a}-\mathrm{c}$

$8 \mathrm{a}-\mathrm{d}$

Reagents: $\boldsymbol{a} . \mathrm{RNH}_{2}$ / trifluoroacetic acid, o-dichlorobenzene; $\boldsymbol{b} . \mathrm{R}_{1} \mathrm{~W} / \mathrm{AcOH}$;

6a $\mathrm{X}=p-\mathrm{C}_{6} \mathrm{H}_{4} ; \mathbf{6 b} \mathrm{X}=m-\mathrm{C}_{6} \mathrm{H}_{4} ; \mathbf{6 c} \mathrm{X}=\left(\mathrm{CH}_{2}\right)_{4} ; \mathbf{7 a} \mathrm{R}=\mathrm{Ph}, \mathrm{X}=p-\mathrm{C}_{6} \mathrm{H}_{4} ; \mathbf{7 b} \mathrm{R}=\mathrm{Ph}, \mathrm{X}=m-\mathrm{C}_{6} \mathrm{H}_{4}$; 7c $\mathrm{R}=p-\mathrm{BrC}_{6} \mathrm{H}_{4}, \mathrm{X}=\left(\mathrm{CH}_{2}\right)_{4}$. 8a $\mathrm{R}=\mathrm{Ph}, \mathrm{R}_{1}=1-\mathrm{Ad}, \mathrm{X}=p-\mathrm{C}_{6} \mathrm{H}_{4} ; \mathbf{8 b} \mathrm{R}=\mathrm{Ph}, \mathrm{R}_{1}=1-\mathrm{Ad}, \mathrm{X}=m$ $\mathrm{C}_{6} \mathrm{H}_{4} ; \mathbf{8 c} \mathrm{R}=\mathrm{Ph}, \mathrm{R}_{1}=\mathrm{t}-\mathrm{Bu}, \mathrm{X}=p-\mathrm{C}_{6} \mathrm{H}_{4} ; \mathbf{8 d ~ R}=p-\mathrm{BrC}_{6} \mathrm{H}_{4}, \mathrm{R}_{1}=1-\mathrm{Ad}, \mathrm{X}=\left(\mathrm{CH}_{2}\right)_{4}$.

\section{Scheme 2}

Further quaternization of bistriazoles 7 by alkylhalogenides proceeds readily in acetic acid to afford the 3,3'-bridged bistriazolium salts $8 \mathrm{a}-\mathrm{d}$ in $75-77 \%$ yields. The use of acetic acid is peculiarly desirable in the cases of the 1-bromoadamantane and tert-butyl iodide reactions, because in aprotic media in the presence of bases results in dehydrohalogenation.

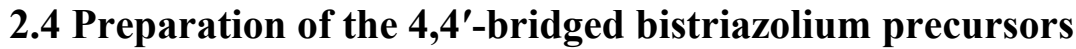

Bistriazoles 9a,b were obtained by the ring transformations of 2-phenyl-1,3,4-oxadiazole 2a with $p$-phenylenediamine hydrochloride in the presence of sodium acetate, and $\mathbf{2 a}$ with $\mathrm{m}$ phenylenediamine in the presence of trifluoroacetic acid in $o$-dichlorobenzene. In turn, $\mathbf{9 a}, \mathbf{b}$ were quaternized with 1-bromoadamantane to afford the 4,4'-bridged bistriazolium salts 10a,b (yields $67-92 \%)^{26,31}$ (Scheme 3). 


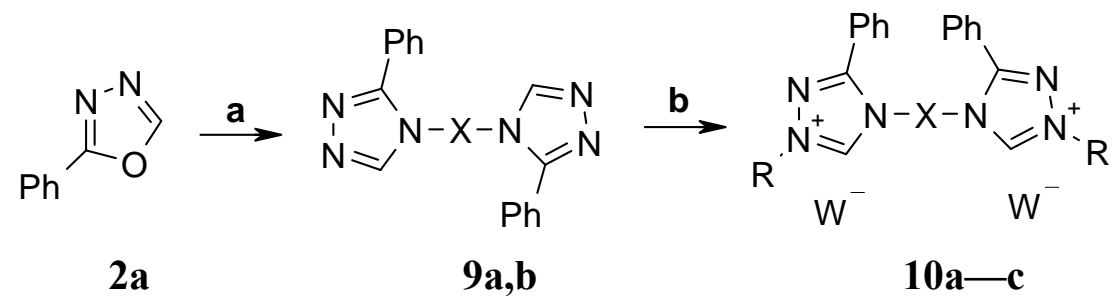

Reagents: $\boldsymbol{a} \mathrm{H}_{2} \mathrm{NXNH}_{2}, \mathrm{H}^{+}$, o-dichlorobenzene; $\boldsymbol{b}$ RHal, AcOH. 9a X $=p$ - $\mathrm{C}_{6} \mathrm{H}_{4}, 9 \mathbf{b} \mathrm{X}=m-\mathrm{C}_{6} \mathrm{H}_{4}$; 10a $\mathrm{R}=1$-Ad; $\mathrm{X}=p-\mathrm{C}_{6} \mathrm{H}_{4} ; 10 b \mathrm{R}=1$ - $\mathrm{Ad} ; \mathrm{X}=m-\mathrm{C}_{6} \mathrm{H}_{4} ; 10 \mathbf{c} \mathrm{R}=t$ - $\mathrm{Bu} ; \mathrm{X}=p-\mathrm{C}_{6} \mathrm{H}_{4} ; \mathbf{1 0 a}-\mathbf{c} \mathrm{W}=$ $\mathrm{Br}, \mathrm{ClO}_{4}$.

\section{Scheme 3}

\subsection{Preparation of the 1,1'-bridged triazolium biscarbene precursors}

The preparation of these compounds was carried out in two stages. In the first stage, alkylation of triazole 2a with 2,2'-dichlorodiethyl ether or 1-bromo-4-chlorobutane produced the monohalogenalkyltriazolium salts 11a,b, which further alkylated the second molecule of triazole $\mathbf{2 a}$ to form the bis-salts $\mathbf{1 2} \mathbf{a}, \mathbf{b}^{31}$. These processes proceeded consecutively and usually it is not necessary to conduct them in separate stages. In the case of the preparation of salt $\mathbf{1 2 b}$, due to the difficulty of separating the side products of quaternization at the 2 position, it was expedient to prepare salt 11b first by the quaternization of 3,4-diphenyl-1,2,4-triazole 2a in the excess of 2,2'dichlorodiethyl ether, and then to perform the second stage with pure salt $\mathbf{1 1} \mathbf{b}$ and triazole $\mathbf{2 a}$.

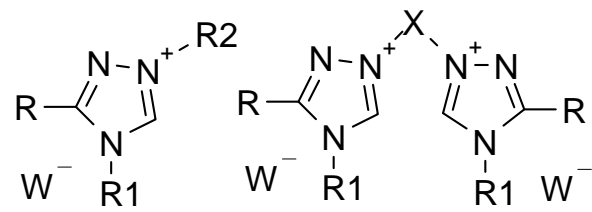

$11 \mathbf{a , b}$

12a,b

11a,12a R $=\mathrm{C}_{6} \mathrm{H}_{5}, \mathrm{R}_{1}=p-\mathrm{BrC}_{6} \mathrm{H}_{4}, \mathrm{X}=\left(\mathrm{CH}_{2}\right)_{4} ; 12 \mathrm{~b} \mathrm{R}=\mathrm{R}_{1}=\mathrm{C}_{6} \mathrm{H}_{5}, \mathrm{X}=\left(\mathrm{CH}_{2}\right)_{2} \mathrm{O}\left(\mathrm{CH}_{2}\right)_{2}$; $12 \mathrm{a}, \mathrm{b} \mathrm{W}=\mathrm{ClO}_{4}$. $11 \mathrm{~b} \mathrm{R}=\mathrm{R}_{1}=\mathrm{C}_{6} \mathrm{H}_{5}, \mathrm{R}_{2}=\left(\mathrm{CH}_{2}\right)_{2} \mathrm{O}\left(\mathrm{CH}_{2}\right)_{2} \mathrm{Cl}$

\subsection{A triazolium precursor with an $\mathrm{OH}$ functional group}

Although the carbenes with functional groups have been obtained in situ ${ }^{14}$ and isolated in a pure state ${ }^{16}$, heteroaromatic carbenic structures with anionic functionalities have not received attention until recently. Such structures, however, are of interest because they imply the possibility of additional stabilization due to the presence of an anionic center in chelated complexes.

The synthetic approach to the desired compounds involved the use of functional hydroxyarylsubstituted triazolium precursors. The synthesis of one such precursor was carried out in two stages (Scheme 4): first, 3-phenyl-4-(2-hydroxyphenyl)-1,2,4-triazole 13 was prepared by the 
ring transformation of 2-phenyl-1,3,4-oxadiazole 2a with $o$-aminophenole in $o$-dichlorobenzene solution, and second, 2) 1-(1-adamantyl)-3-phenyl-4-(2-hydroxyphenyl)-1,2,4-triazolium bromide $14\left(\mathrm{X}=\mathrm{Br}, \mathrm{ClO}_{4}\right)$ was prepared by the quaternization of triazole 13 with 1bromoadamantane in acetic acid.

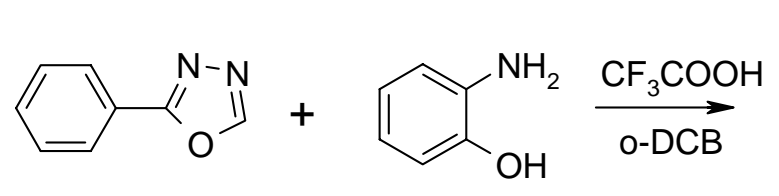

$2 \mathbf{a}$<smiles>Oc1ccccc1-n1cnnc1-c1ccccc1</smiles>

13<smiles>[X]c1ccccc1-n1c[n+]([Al])nc1-c1ccc(C(=O)O)cc1</smiles>

14

\section{Scheme 4}

\section{Heteroaromatic carbenes of the 1,2,4-triazole and benzimidazole series}

In order to achieve an accelerated rate of carbene formation and also to simplify the product isolation procedure, we have examined the influence of solvents (aromatic solvents and acetonitrile) on the process of deprotonation of imidazolium, benzimidazolium, and 1,2,4triazolium salts. Aromatic solvents proved to be convenient for the isolation of the desired carbenes, and acetonitrile was found to accelerate the rate of carbene formation due to the high polarity of this solvent ( $\mu 3,37 \mathrm{D})$.

\subsection{Monocarbenes of the 1,2,4-triazole series}

In the course of this work, we came across a very convenient method for the preparation of carbenes by deprotonation of triazolium salts 1a,d,e with potassium tert-butoxide in aromatic solvents (method A) ${ }^{27}$. In this method it is necessary that the reagent be sufficiently soluble in benzene and toluene (for potassium tert-butoxide about $2 \%$ at room temperature), in order to facilitate rapid reactions with salt in dispersions. 


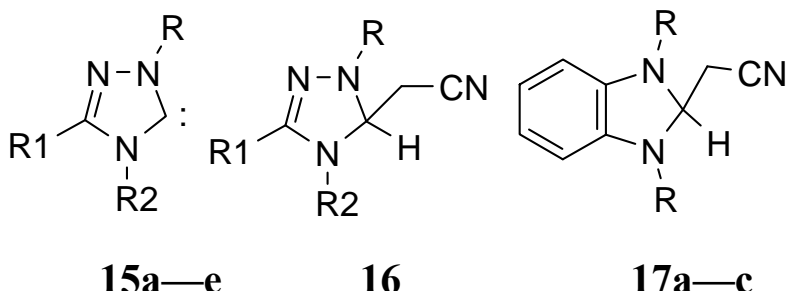

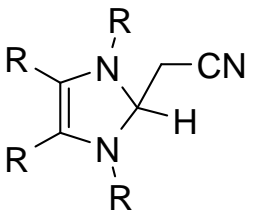

18

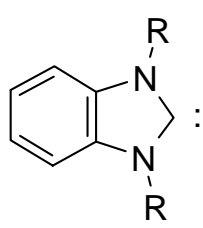

19

15a-d,16 $\mathrm{R}=1-\mathrm{Ad} ; \mathbf{1 5 e} \mathrm{R}=t$ - $\mathrm{Bu} ; \mathbf{1 5 a}, 16 \mathrm{R}_{1}=\mathrm{R}_{2}=\mathrm{C}_{6} \mathrm{H}_{5} ; 15 \mathbf{b} \mathrm{R}_{1}=\mathrm{C}_{6} \mathrm{H}_{5}, \mathrm{R}_{2}=p-\mathrm{BrC}_{6} \mathrm{H}_{4} ; \mathbf{1 5 c}$ $\mathrm{R}_{1}=\mathrm{R}_{2}=p-\mathrm{BrC}_{6} \mathrm{H}_{4} ; 15 d \mathrm{R}_{1}=\mathrm{C}_{6} \mathrm{H}_{5}, \mathrm{R}_{2}=1-\mathrm{C}_{10} \mathrm{H}_{7} ; 17 \mathbf{a} \mathrm{R}=\mathrm{CH}_{3} ; 17 \mathbf{b} \mathrm{R}=\mathrm{CH}_{2} \mathrm{C}_{6} \mathrm{H}_{5} ; 17 \mathbf{c}, 19 \mathrm{R}=$ $1-\mathrm{Ad} ; 18 \mathrm{R}=\mathrm{C}_{6} \mathrm{H}_{5}$.

The modification of the deprotonation process enabled us to prepare adequately pure samples of 1-alkyl-3,4-diaryl-1,2,4-triazol-5-ylidenes 15a,d,e in good yields (64, 51 and 82\%, respectively). In the first stage of the reaction, an ion exchange to the tert-butoxide salt takes place. The second stage comprises decomposition of the azolium tert-butoxide into carbene and tert-butanol. In some cases (1a), the azolium tert-butoxides are isolable and only slowly decompose under heating in toluene solution. The process is assisted by the presence of alcohols (methanol, isopropanol or tert-butanol) in toluene solution. Upon removal of the solvent, most of the azolium alkoxide decomposes to the corresponding carbene and the latter can be purified by recrystallization. Owing to the simplicity of the process and the use of an easily dried, nonaqueous solvent, approach $\mathrm{A}$ is a very convenient method for the synthesis of various types of HC. This method can be universal because it is almost never accompanied by any side reactions. Furthermore, as compared to the method of Arduengo et al. ${ }^{5,6}$, the new modification avoids two additional preparative stages, namely removal of THF and extraction of the carbene with benzene.

The reactions of sodium hydride with salts $\mathbf{1 a - c}$ in acetonitrile solution also proceed satisfactorily and lead directly to stable carbenes, 1,2,4-triazol-5-ylidenes 15a-c (procedure B) that are deposited as colorless precipitates ${ }^{27}$. However, 1,2,4-triazol-5-ylidene 15a, which was prepared in situ, underwent partial insertion into the $\mathrm{C}-\mathrm{H}$ bond of acetonitrile upon heating or on prolonged storage at room temperature to form a low yield (18\%) of azoline 16. The same result was obtained by the interaction of pure 1,2,4-triazol-5-ylidene 15a with acetonitrile. The reaction was carried out at $100{ }^{\circ} \mathrm{C}$ for $10 \mathrm{~h}$ (azoline 16 was produced in approximately $50 \%$ yield according to the NMR data but it was isolated in only $30 \%$ yield). This insertion proceeds as a consecutive reaction after formation of the carbene from salt 1a by treatment with sodium hydride $(0.5 \mathrm{~h})$. This side-reaction results in a decreased yield of carbene. For example, the yield of $15 \mathbf{a}$ is $40 \%$. On the other hand, carbenes $15 \mathbf{b}, \mathbf{c}$ were obtained in high yields (68-95\%) without perceptible amounts of the azolines of type $\mathbf{1 6 .}$

Comparing methods $\mathrm{A}$ and $\mathrm{B}$, it should be noted that the procedure A sometimes gives better results than procedure $B$, particularly for the synthesis of 15a. Interestingly, it was not possible to synthesize 15d by method B. 
In comparison with the method of Enders et al. ${ }^{8}$, procedure B permits the synthesis of 1,2,4triazol-5-ylidenes $\mathbf{1 5}$ in one stage in toluene solution with minimal expense and preparation. These carbenes probably have smaller nucleophilicities than those of imidazol-2-ylidenes and can therefore be considered as ambident compounds (one cyclic nitrogen atom $\mathrm{N}^{2}$ and a carbon atom $\mathrm{C}^{5}$ have free electron pairs in the plane of a heterocyclic ring).

\subsection{A monocarbene of the benzimidazole series}

When we attempted the synthesis of 1,3-di(1-adamantyl)-benzimidazol-2-ylidene in THF solution using the method of Arduengo et al. ${ }^{5,6}$, it was found that the sodium hydride procedure was very slow ( $>1$ day) ${ }^{34}$. We were therefore prompted to search for a more convenient preparation for this carbene.

Specifically, we studied the deprotonation of the 2-unsubstituted benzimidazolium salts 4a-c and imidazolium salt $\mathbf{5}$ in anhydrous acetonitrile using procedure B. The reaction with sodium hydride resulted in the products of insertion of the corresponding carbenes into the $\mathrm{C}-\mathrm{H}$ bond of acetonitrile, namely 2 -cyanomethyl- $2 H$-azolines $\mathbf{1 7 a - c , 1 8}{ }^{27}$. These compounds were isolated in a pure crystalline state for the first time. The $\mathrm{C}-\mathrm{H}$ insertion of carbenes in acetonitrile also proceeds by deprotonation of the sterically hindered 1,3-(1-adamantyl)benzimidazolium salt 4c, in which a $\mathrm{C}^{2} \mathrm{H}$ link is shielded by bulky adamantyl substituents. Compound $\mathbf{1 7} \mathbf{c}$ is not a solvate similar to Arduengo's compound obtained from 1,3-di(1-adamantyl)imidazol-2-ylidene and acetonitrile ${ }^{35}$. This is a polarized form of the cyanomethyl derivative of azoline as confirmed by a slightly elongated bond $\mathrm{C}^{2}-\mathrm{CH}_{2}$ (for $\mathbf{1 7 c} 154.8 \mathrm{pm}$ versus $153.5 \mathrm{pm}$ for $\mathbf{1 8}$ ). The structures of carbenes will be discussed in more detail below.

The formation of azolines in these reactions can result from proton elimination from an azolium salt to form carbene (Crb), further proton elimination by the carbene from acetonitrile, and recombination of the intermediate azolium cation with cyanomethanide anion $\left[\mathrm{CH}_{2} \mathrm{CN}\right]^{-}$or a concerted pathway for the reaction.

$$
\mathbf{4}+\mathrm{NaH} \rightarrow \mathbf{C r b} ; \quad \mathbf{C r b}+\mathrm{CH}_{3} \mathrm{CN} \rightarrow \mathbf{1 7}
$$

An alternative mechanism for this reaction is initial generation of the anion $\left[\mathrm{CH}_{2} \mathrm{CN}^{-}\right.$ followed by recombination with an azolium cation. However, this mechanism is not feasible because the reaction of acetonitrile with sodium hydride proceeds much slower in comparison to that with azolium salts (as observed by the TLC method), which can be explained by the smaller acidity of acetonitrile $\left(\mathrm{p} K_{\mathrm{a}} 25\right)$ compared with that of azolium salts $\left(\mathrm{p} K_{\mathrm{a}}<24\right)^{36,37}$.

In order to elucidate the details of what pathway really occurs, we carried out the reaction of acetonitrile with 2-deuterium-1,3-dibenzylbenzimidazolium chloride in the presence of sodium hydride (Scheme 5). The absence of deuterium in the isolated 2-cyanomethylazoline $\mathbf{1 7 b}$ supports the carbene mechanism of the reaction (which is most likely a concerted process in accord with the Moss concept of the intermediate mechanism between nucleophilic and electrophilic ${ }^{38}$ ). 


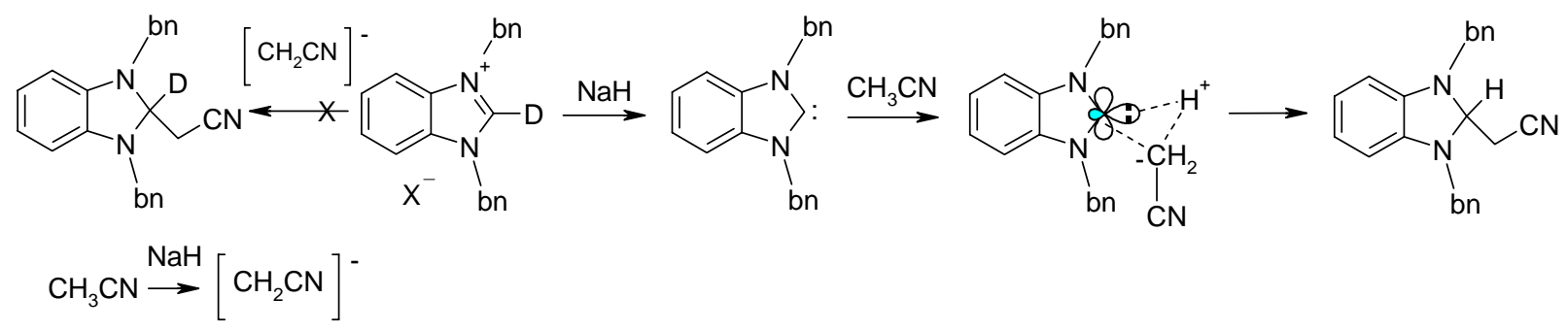

4b

$17 \mathbf{b}$

\section{Scheme 5}

It should be noted that under comparable conditions acyclic phosphanylsilylcarbenes react with acetonitrile to form azirines ${ }^{39}$, and the reaction of electrophilic $N$-fluoropyridylidene with nitriles and cyanates in situ affords pyrido[1,2-a]-1,3,5-triazin-4-ones ${ }^{40}$.

As mentioned above, sterically hindered compound 17c exhibits enhanced stability upon prolonged storage and does not undergo reaction below $100{ }^{\circ} \mathrm{C}$ in a solid state. However, heating solid $17 \mathrm{c}$ at $180{ }^{\circ} \mathrm{C}$ results in $\alpha$-elimination of acetonitrile to afford a stable carbene 19 (procedure C) ${ }^{27 a}$. Heating this compound in aromatic solvents (benzene or toluene) gives analogous results. However, more time is required for the process. Thus, for the first time a stable representative of the benzimidazole carbene series has been produced as a colorless highmelting solid that can be crystallized from toluene and stored at room temperature under argon for at least six months without decomposition.

We assume that the synthesis of carbenes via the intermediate cyanomethylazolines (procedure C) can be employed for other sterically-hindered compounds that are similar to 19. This point will be studied later. However, the elimination of acetonitrile from sterically open compounds $\mathbf{1 7 a} \mathbf{a}, \mathbf{b}, \mathbf{d}$ failed. Most likely, the strength of Ht-C bonds turned out to be much greater than those in azoline 17c, and their decomposition requires high temperatures thus causing other side reactions to occur.

The reverse reaction to the decomposition $17 \mathrm{c} \rightarrow \mathbf{1 9}$, i.e. the insertion $19 \rightarrow \mathbf{1 7 c}$, proceeds almost instantly (the conversion of carbene $\mathbf{1 9}$ in acetonitrile is complete in a few min at room temperature and in 15-30 min in toluene solution).

Thus, we have found that the insertion into a $\mathrm{C}-\mathrm{H}$ bond of acetonitrile proceeds effectively with highly basic HC (benzimidazol-2-ylidenes and aromatic-substituted imidazol-2-ylidenes). However, this reaction takes place even with 1,2,4-triazol-5-ylidenes albeit at a much slower rate. We have also synthesized a new type of $\mathrm{HC}$, namely benzimidazol-2-ylidene 19, and a series of new 1,2,4-triazol-5-ylidenes 15a-e with different substituents (adamantyl, tert-butyl, aryl), which are very stable upon prolonged storage. These compounds are also stable to oxygen and relatively stable to water in comparison with the usual (alkyl, aryl) substituted azolylidenes.

It should be noted that independently from our work, and almost simultaneously, Hahn and co-workers reported a representative of the related carbene 1,3-di-neopentylbenzimidazol-2ylidene by the reduction of the corresponding thione with sodium-potassium alloy at room 
temperature for 20 days ${ }^{41}$. This interesting carbene exists in two chiral forms. From the aforementioned description it would appear that our synthesis of 19 synthetic method ${ }^{27 a}$ is somewhat simpler. Moreover, our product only features one isomer, which is more stable than the carbene described by Hahn et al.

\subsection{Heteroaromatic biscarbenes of the 1,2,4-triazole series}

The high stability of 1,2,4-triazol-5-ylidenes stimulated our interest in compounds with two carbene-bearing nuclei. Such compounds could serve as monomers for the construction of oligomeric and even polymeric compounds. Moreover, it should be possible to join the carbenic centers by moieties that are capable of conjugation. Polymer structures of this type are anticipated to exhibit enhanced polarizabilities and conductivities as well as interesting magnetic properties. It is known that anisotropic conductivity of electronic effects is associated with heterocyclic compounds ${ }^{42}$, hence it is of special interest to explore such effects through the $1,1^{\prime}$-, $3,3^{\prime}$ and 4,4'-positions of triazolylidene heteronuclei.

Considerable interest has been generated in the chemistry of metal complexes of polycarbenes with conjugated intercarbene bonds as a separate type of the metal-complex structures. The first attempt to obtain such complexes was carried out by Bertrand and co-authors 43 by treatment of 1,2,4-trimethyl-1,2,4-triazolium biscation bistriflate with silver acetate. However, the polymer obtained was not homogenous, stable only in a crystalline state, and the silver ions underwent exchange in solution. Caballero et al. ${ }^{17}$ reported a silver complex of bisimidazol-2-ylidene with a pyridine bridge; however, this complex is not polymeric. While the silver complexes described by Chen et al. ${ }^{44}$ and Melaiye et al. ${ }^{45}$ are oligomeric clusters, these complexes do not involve conjugation. Accordingly, the synthesis of stable metal complexes of polycarbenes remains a synthetic challenge.

For the synthesis of stable 1,2,4-triazole series biscarbenes ${ }^{31}$, the deprotonation of salts $\mathbf{8 a}-\mathbf{c}$, 10a,b and 12b was carried out by treatment with potassium tert-butoxide in aromatic solvents, preferably in toluene (according to the method A), or in mixtures of toluene with alcohols under non-aqueous conditions according to the method described earlier ${ }^{27 a}$. The products of these reactions were the 3,3'-, 4,4'- and 1,1'-bridged biscarbenes $\mathbf{2 0 a - c , ~ 2 1 a , b}$ and 22 in yields 56-85, $50-52$ and $62 \%$, respectively. However, in the latter case carbene 22 formed a complex with potassium perchlorate probably due to a strong chelate interaction with the potassium cation. 
<smiles></smiles>

$20 a-c$<smiles></smiles>

$21 \mathbf{a}, \mathbf{b}$<smiles></smiles>

22

20a $\mathrm{R}=1-\mathrm{Ad}, \mathrm{R}_{1}=\mathrm{Ph}, \mathrm{X}=p-\mathrm{C}_{6} \mathrm{H}_{4} ; 20 b \mathrm{R}=1-\mathrm{Ad}, \mathrm{R}_{1}=\mathrm{Ph}, \mathrm{X}=m-\mathrm{C}_{6} \mathrm{H}_{4} ; 20 \mathbf{c} \mathrm{R}=1-\mathrm{Ad}, \mathrm{R}_{1}=p$ $\mathrm{BrC}_{6} \mathrm{H}_{4}, \mathrm{X}=\left(\mathrm{CH}_{2}\right)_{4}$. 21a $\mathrm{R}=1-\mathrm{Ad} ; \mathrm{X}=p-\mathrm{C}_{6} \mathrm{H}_{4} ; 21 \mathbf{b} \mathrm{R}=1-\mathrm{Ad} ; \mathrm{X}=m-\mathrm{C}_{6} \mathrm{H}_{4} ; 22 \mathrm{R}=\mathrm{R}_{1}=\mathrm{C}_{6} \mathrm{H}_{5}$, $\mathrm{X}=\left(\mathrm{CH}_{2}\right)_{2} \mathrm{O}\left(\mathrm{CH}_{2}\right)_{2}$

Use of the acetonitrile procedure (B), followed by cooling of the reaction mixture at $-40{ }^{\circ} \mathrm{C}$ and subsequent warming to room temperature, resulted in a $63 \%$ yield of biscarbene $\mathbf{2 0 b}$. Highpurity 21a (without traces of alkoxides in the unpurified compound) was prepared via the reaction of potassium hexamethylsilazanide with salt 10a in toluene solution (modified method A).

\subsection{Stable chelate anionocarbene complexes}

The first example of the chelate anionocarbene potassium complex based on the 4-(2hydroxyphenyl)substituted 1,2,4-triazolium salt $\mathbf{1 4}$ has been obtained. Deprotonation of 14 was effected by treatment with potassium tert-butoxide in aromatic solvents, in acetonitrile, dioxane, or THF. The anionocarbene entity $\mathbf{2 3 A}$ was stabilized by interaction with a potassium cation as indicated in $23^{46}$.

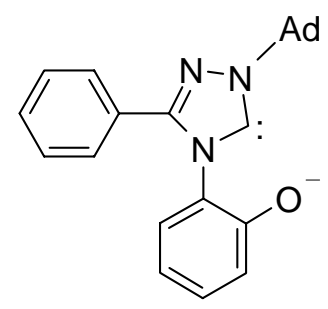

23A

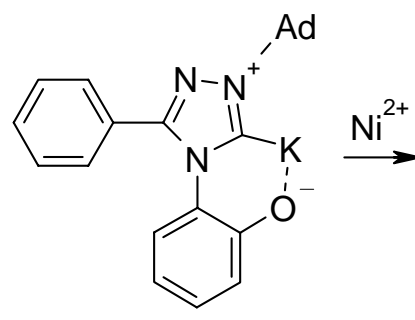

23

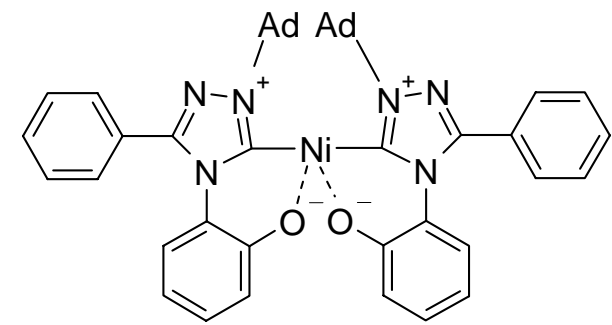

24

\section{Scheme 6}

The formation of alkali metal carbene complexes has been reported by Arduengo and coworkers ${ }^{47}$ e.g. via the reaction of lithium phenolate with 1,3-dimesitylimidazol-2-ylidene. Furthermore, Alder and co-workers ${ }^{13}$ have reported the synthesis of 1,3-diisopropylpyrimidin-2ylidene from the corresponding salt and metal hexamethylsilazanides. However, in both cases relatively unstable bimetallic compounds were formed (see, e.g., A). Lithium azoles $\mathbf{B}^{48}$ are substances with high reactivities that self-associate in dimeric to tetrameric compositions. 
Azolioboranides $\mathbf{C}^{49}$ also deserve mention in this context. The authors of these type B compounds involve lithium bonding to the nitrogen and carbon atoms while in compounds of type $\mathbf{C}$, the lithium is bonded to carbon and boron atoms.

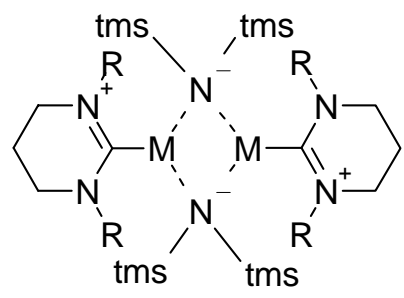

A $(\mathrm{M}=\mathrm{Li}, \mathrm{Na}, \mathrm{K})$

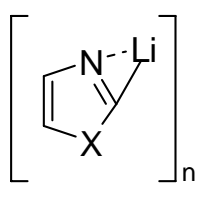

B

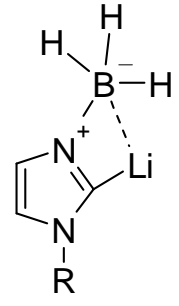

C

A study of the properties of compound $\mathbf{2 3}$ should therefore provide definitive information regarding the structures of these new complexes. It has been established that upon treatment with heavy metal salts, compound $\mathbf{2 3}$ is easily transformed into anionocarbenic heavy metal complexes. This exchange reaction (Scheme 6) was carried out with the help of nickel perchlorate, which affords a nickel bischelate anionocarbene complex, 24. Evidently, this process involves some dissociation of complex $\mathbf{2 3}$ and a shift of the equilibrium to nickel complex 24 due to the low solubility of potassium perchlorate in acetonitrile solution.

\section{Structures of heteroaromatic carbenes, salts and 2(5) $\mathrm{H}$-azolines}

The structures of carbenes $\mathbf{1 5 b}, \mathbf{c}$ and 19 were determined by X-ray analysis and compared with those of salts $\mathbf{1 c}, \mathbf{4 c}$ and $2(5) H$-azolines $\mathbf{1 6 , 1 7 b , c , 1 8}$.

The results of the X-ray analysis of $\mathbf{1 5 b , c}$ (Table 1) reveal an almost planar structure for the carbenic cycles and a significant elongation of the bonds at the carbene centre $r\left(\mathrm{C}^{5}-\mathrm{N}^{1(4)}\right)$ in comparison with that of the corresponding azolium salt 1c where this bond length is between those of single and double bonds. The bond lengths $\mathrm{C}^{5}-\mathrm{N}^{1}(134-135 \mathrm{pm})$ and $\mathrm{C}^{5}-\mathrm{N}^{4}$ (approximately $138.5 \mathrm{pm}$ ) in $\mathbf{1 5 b}, \mathbf{c}$ are somewhat shorter than those of single $\mathrm{C}-\mathrm{N}$ bonds (e.g., $\mathrm{r}$ 148-149 pm for $\left.\mathrm{N}^{1}-\mathrm{C}^{\mathrm{Ad}}\right)$. In the structure of salt $1 \mathrm{c}$ the same bonds are also delocalized but are closer in length to that of a typical double bond ( 132,$2 ; 133,6 \mathrm{pm}$ ). Interestingly, the largest interaction with a heteronucleus for the substituent at the atom $\mathrm{N}^{4}$ occurs in azoline $16\left(\mathrm{r} \mathrm{N}^{4}-\mathrm{C}^{30}\right.$ $141.9 \mathrm{pm})$ and is less in the corresponding carbene (143.5-144 pm) and salt (145.1 pm). The last observation cannot be explained by the dihedral angle differences between the aromatic rings and the heteronucleus in these compounds. In the case of azoline $\mathbf{1 6}$ the dihedral angle is larger than that for salt 1c; however, the interaction is the largest. In contrast, the differences in the $C^{3}$ substituent influence are almost undetectable, as is evident from the close values of the $\mathrm{C}^{3}-\mathrm{C}^{20}$ bond lengths in azoline 16, salt 1c, and carbenes 15b,c. The interaction of the adamantyl 
substituent with the heteronucleus in carbenes $\mathbf{1 5 b}, \mathbf{c}$ is somewhat larger than that in azoline $\mathbf{1 6}$ and salt $1 \mathbf{c}$.

The reduced bond lengths in carbenes $15 b, c$ relative to those of azoline 16 and $\mathrm{C}^{3}=\mathrm{N}^{2}$ bond elongation, which is a normal double bond in azoline, implies electron delocalization in these carbenes and is consistent with their aromatic character.

Table 1. Selected bond lengths $(\mathrm{pm})$, internal $(\theta)$ and dihedral $(\varphi)$ angles (degrees) for compounds $\mathbf{1 c}, \mathbf{1 5 b}, \mathbf{c}, 16^{\mathrm{a}}$ relative to the known data for $15^{8}$

\begin{tabular}{cccccc}
\hline Property & $\mathbf{1 c}$ & $\mathbf{1 5 b}$ & $\mathbf{1 5 c}$ & $\mathbf{1 5}^{\mathrm{b}}$ & $\mathbf{1 6}^{\mathrm{c}}$ \\
\hline $\mathrm{r}\left(\mathrm{C}^{5}-\mathrm{N}^{1}\right)$ & $131.2(4)$ & $134.6(3)$ & $134.6(5)$ & $135.1(3)$ & $148.5(4)$ \\
$\mathrm{r}\left(\mathrm{C}^{5}-\mathrm{N}^{4}\right)$ & $132.9(4)$ & $138.6(3)$ & $138.4(5)$ & $137.3(4)$ & $147.1(4)$ \\
$\mathrm{r}\left(\mathrm{N}^{1}-\mathrm{N}^{2}\right)$ & $136.7(4)$ & $139.4(3)$ & $138.8(6)$ & $139.8(3)$ & $144.4(4)$ \\
$\mathrm{r}\left(\mathrm{C}^{3}=\mathrm{N}^{2}\right)$ & $131.5(4)$ & $130.1(3)$ & $130.0(5)$ & $130.4(3)$ & $128.2(4)$ \\
$\mathrm{r}\left(\mathrm{C}^{3}-\mathrm{N}^{4}\right)$ & $138.1(4)$ & $138.4(3)$ & $138.4(5)$ & $139.1(2)$ & $140.5(4)$ \\
$\mathrm{r}\left(\mathrm{N}^{1}-\mathrm{C}^{10}\right)$ & $149.3(4)$ & $148.0(3)$ & $148.1(5)$ & - & $149.4(4)$ \\
$\mathrm{r}\left(\mathrm{C}^{3}-\mathrm{C}^{20}\right)$ & $146.7(4)$ & $147.3(3)$ & $147.8(5)$ & - & $147.6(5)$ \\
$\mathrm{r}\left(\mathrm{N}^{4}-\mathrm{C}^{30}\right)$ & $145.1(5)$ & $143.5(3)$ & $144.0(5)$ & - & $141.9(4)$ \\
$\theta\left(\mathrm{N}^{1}-\mathrm{C}^{5}-\mathrm{N}^{4}\right)$ & $107.6(3)$ & $100.3(2)$ & $100.3(3)$ & $100.6(2)$ & $103.9(3)$ \\
$\varphi\left(\mathrm{C}^{5}-\mathrm{N}^{1}-\mathrm{N}^{2}-\mathrm{C}^{3}\right)$ & $-0.1(1)$ & $-0.2(2)$ & $1.7(5)$ & - & $11.8(4)$ \\
$\varphi\left(\mathrm{N}^{4}-\mathrm{C}^{3}-\mathrm{C}^{20}-\mathrm{C}^{21}\right)$ & $-30.8(4)$ & $43.2(3)$ & $-25.4(7)$ & - & $39.8(5)$ \\
$\varphi\left(\mathrm{C}^{3}-\mathrm{N}^{4}-\mathrm{C}^{30}-\mathrm{C}^{31}\right)$ & $104.3(4)$ & $-125.8(2)$ & $-72.9(5)$ & - & $-173.7(3)$ \\
\hline
\end{tabular}

Note. ${ }^{\text {a }}$ The numbering system for the atoms in a heteronucleus here and in Table 2 corresponds to the protocol used in the chemical name of a compound. $\mathrm{C}^{10}, \mathrm{C}^{20}$, etc are the atoms of the substituents adjacent to the heteronucleus. The carbon atoms of the fragment $\mathrm{CH}_{2} \mathrm{CN}$ are $\mathrm{C}^{6}, \mathrm{C}^{7}$. ${ }^{\mathrm{b}} \mathbf{1 5}\left(\mathrm{R}=\mathrm{R}_{1}=\mathrm{R}_{2}=\mathrm{C}_{6} \mathrm{H}_{5}\right){ }^{8} ;{ }^{\mathrm{c}} \mathrm{r}\left(\mathrm{C}^{2}-\mathrm{C}^{6}\right) 153.3(5) ;{ }^{\mathrm{d}} \mathrm{cf} . \varphi\left(\mathrm{C}^{5}-\mathrm{N}^{4}-\mathrm{C}^{3}-\mathrm{N}^{2}\right) 0.5(5)$

The results of the X-ray analysis of $\mathbf{1 9}$ (Table 2) also reveal a significant elongation of bonds at the carbene centre $\mathrm{r}\left(\mathrm{C}^{2}-\mathrm{N}^{1(3)}\right)$ in comparison with that of the corresponding azolium salt $4 \mathbf{c}$ which possesses bond lengths intermediate between those of single and double bonds (approximately $137 \mathrm{pm}$ for 19). These bonds are essentially shorter than those of single $\mathrm{C}-\mathrm{N}$ bonds (e.g., $\mathrm{r} 149-150 \mathrm{pm}$ for $\mathrm{N}^{1(3)}$-substit.)]. All the bonds of heteronucleus 19 are shorter than those of azoline 17c due to the increase of electron delocalization in the carbene. In the structure of salt $4 \mathbf{c}$ the same bonds are also delocalized; however the pertinent distances are closer to that of a typical double bond ( $132.2 ; 133.6 \mathrm{pm}$ ).

The benzimidazole ring in $\mathbf{1 9}$ is almost planar in order to facilitate conjugation. The reduction of $\mathrm{N}^{1}-\mathrm{C}^{10}$ bond length in proceeding from model 1,3-di(1-adamantyl)benzimidazol-2thione to azoline 17c with further decreases to carbene 19 and salt $4 \mathbf{c}$ is probably due to reduction of steric hindrance at the $\mathrm{C}^{2}$ atom and also to electron withdrawal effects. The structures of the azolines $\mathbf{1 7 b}, \mathbf{c}, \mathbf{1 8}$ each involve an almost planar $\mathrm{N}^{3}-\mathrm{C}^{4}-\mathrm{C}^{5}-\mathrm{N}^{1}$ fragment, and the 
atom $\mathrm{C}^{2}$ deviates $5-19^{\circ}$ out of the plane. Unusual structural characteristics are exhibited by azoline $17 \mathbf{b}$ in the sense that the $\mathrm{C}^{5}-\mathrm{N}^{1}$ bond length is close to the aromatic value (139.4 pm), i.e: the $\mathrm{C}^{2}-\mathrm{N}^{1}$ bond length is essentially shortened by 1-2 pm in comparison with that of azolines $\mathbf{1 7} \mathbf{c}, \mathbf{1 8}$. In contrast to the latter, the heteronucleus of $\mathbf{1 7 b}$ is approximately symmetrical with respect to the plane perpendicular to the $N^{3}-C^{4}-C^{5}-N^{1}$ fragment, $C^{2}$, and the middle of $C^{4}=C^{5}$ bond, and it exhibits the smallest deviation from the plane between azolines 16,17b,c,18 $\left[\varphi\left(\mathrm{C}^{2}-\right.\right.$ $\left.\mathrm{N}^{1}-\mathrm{C}^{5}-\mathrm{C}^{4}\right) 5.2^{\circ}$. The $\mathrm{N}^{1}-\mathrm{C}^{10}$ bond length of 146.3 is substantially smaller than $\mathrm{N}-\mathrm{C}$ bond lengths of aliphatic fragments. However, the $\mathrm{N}^{1}-\mathrm{C}^{10}$ bond length is similar to those of benzylsubstituted compounds with an electron-withdrawing nucleus (e.g. $146.5 \mathrm{pm}$ for 1,3dibenzylbenzimidazol-2-thione).

Table 2. Selected bond lengths (pm), internal $(\theta)$ and dihedral $(\varphi)$ angles (degrees) for compounds $4 \mathbf{c}, \mathbf{1 7 b}, \mathbf{c}, 18,19^{\mathrm{a}}$ relative to those for thiones ${ }^{\mathrm{b}, \mathrm{c}}$ (the authors data)

\begin{tabular}{cccccccc}
\hline Property & $\mathbf{4 c}$ & $\mathbf{1 7 b}$ & $\mathbf{1 7 c}$ & $\mathbf{1 8}$ & $\mathbf{1 9}$ & $\mathrm{b}$ & $\mathrm{c}$ \\
\hline $\mathrm{r}\left(\mathrm{C}^{2}-\mathrm{N}^{1}\right)$ & $133.6(4)$ & $145.9(2)$ & $147.6(3)$ & $146.6(3)$ & $137.4(4)$ & $138.9(2)$ & $136.7(2)$ \\
$\mathrm{r}\left(\mathrm{C}^{5}-\mathrm{N}^{1}\right)$ & $139.9(4)$ & $139.4(2)$ & $143.9(3)$ & $145.3(3)$ & $140.2(4)$ & $140.4(2)$ & $139.0(2)$ \\
$\mathrm{r}\left(\mathrm{C}^{5}-\mathrm{C}^{4}\right)$ & $141.5(4)$ & $140.4(2)$ & $141.1(3)$ & $136.4(3)$ & $139.8(4)$ & $140.3(3)$ & $139.8(3)$ \\
$\mathrm{r}\left(\mathrm{N}^{1}-\mathrm{C}^{10}\right)$ & $148.9(4)$ & $146.3(2)$ & $150.7(3)$ & $143.0(3)$ & $149.3(4)$ & $152.1(2)$ & $146.5(2)$ \\
$\theta\left(\mathrm{N}^{1}-\mathrm{C}^{2}-\right.$ & $112.2(3)$ & $103.7(1)$ & $106.0(2)$ & $105.0(2)$ & $103.8(2)$ & $108.1(1)$ & $106.4(2)$ \\
$\left.\mathrm{N}^{3}\right)$ & & & & & & & \\
$\theta\left(\mathrm{C}^{2}-\mathrm{N}^{1}-\right.$ & $107.6(2)$ & $109.0(1)$ & $102.4(2)$ & $104.5(2)$ & $112.2(2)$ & $108.3(1)$ & $110.3(1)$ \\
$\left.\mathrm{C}^{5}\right)$ & & & & & & & $106.8(1)$ \\
$\theta\left(\mathrm{N}^{1}-\mathrm{C}^{5}-\right.$ & $106.9(2)$ & $108.6(1)$ & $110.4(2)$ & $108.9(2)$ & $105.8(2)$ & $107.8(1)$ & $106.5(1)$ \\
$\left.\mathrm{C}^{4}\right)$ & & & & & & & \\
$\varphi\left(\mathrm{C}^{2}-\mathrm{N}^{1}-\right.$ & $0.2(6)$ & $5.2(2)$ & $17.0(2)$ & $18.8(3)$ & $0.5(3)$ & - & - \\
$\left.\mathrm{C}^{5}-\mathrm{C}^{4}\right)$ & & & & &
\end{tabular}

Note. ${ }^{a} \mathrm{C}^{10}, \mathrm{C}^{20}$, etc are the atoms of the substituents adjacent to the heteronucleus. Some other pertinent bond lengths and angles: $4 \mathbf{c} r\left(C^{2}-\mathrm{N}^{3}\right)$ 132.2(4); $r\left(\mathrm{C}^{4}-\mathrm{N}^{3}\right)$ 139.6(4); $\mathrm{r}\left(\mathrm{N}^{3}-\mathrm{C}^{20}\right) 150.0(4)$; $17 \mathrm{c} \mathrm{r}\left(\mathrm{C}^{2}-\mathrm{C}^{6}\right)$ 154.8(3); $\mathrm{r}\left(\mathrm{C}^{4}-\mathrm{N}^{3}\right)$ 139.6(3); $\varphi\left(\mathrm{C}^{2}-\mathrm{N}^{1}-\mathrm{C}^{5}-\mathrm{C}^{4}\right)$ 17.0(2); $\varphi\left(\mathrm{N}^{1}-\mathrm{C}^{2}-\mathrm{C}^{6}-\mathrm{C}^{7}\right) 65.0$ (2). $19 \mathrm{r}\left(\mathrm{C}^{2}-\mathrm{N}^{3}\right)$ 137.2(4); $\mathrm{r}\left(\mathrm{C}^{4}-\mathrm{N}^{3}\right)$ 139.9(4); $\mathrm{r}\left(\mathrm{N}^{3}-\mathrm{C}^{20}\right)$ 149.4(3).

${ }^{\mathrm{b}}$ 1,3-di(1-adamantyl)benzimidazol-2-thione $\mathrm{r}\left(\mathrm{C}^{2}-\mathrm{S}\right)$ 168.05(19); ${ }^{\mathrm{c}}$ 1,3-dibenzylbenzimidazol-2thione $\mathrm{r}\left(\mathrm{C}^{2}-\mathrm{S}\right) 168.0(2)$.

The internal angles in the carbene heteronuclei of $\mathbf{1 5 b}, \mathbf{c}, \mathbf{1 9}$ are reminiscent of those at the pyridine nitrogen atoms of azoles. Thus, the $\theta\left(\mathrm{N}^{1}-\mathrm{C}^{2}-\mathrm{N}^{3}\right)$ angle at the carbenic carbon atom in 19 is $103.8^{\circ}$, which is much smaller than that for salt $\mathbf{4 c}$. Analogous changes were found upon comparison of the angles at the $\mathrm{C}_{5}$ atom $\theta\left(\mathrm{N}^{1}-\mathrm{C}^{5}-\mathrm{N}^{4}\right)$ for salt $1 \mathbf{c}$ and carbenes $\mathbf{1 5 b}$,c. The difference between carbenes 19 and $\mathbf{1 5 b}, \mathbf{c}$ is clearly evident from the angle differences at the carbene carbon atom $\theta(\mathrm{N}-\mathrm{C}-\mathrm{N})$, which for carbene $19\left(103.8^{\circ}\right)$ is appreciably larger than those 
for 1,2,4-triazol-5-ylidenes $\mathbf{1 5 b}, \mathbf{c}\left(100.3^{\circ}\right)$ and intermediate between those for imidazol-2ylidenes $\left(101-102^{\circ}\right)^{5,6}$ and imidazolin-2-ylidenes $\left(104.7-106.4^{\circ}\right)^{50}$. It appears that the internal angle at a carbene carbon atom can be used as an indicator of stabilization of the nucleophilic cyclic carbenes at the expense of aromatic delocalization.

Based on the experimental parameters for delocalization, such as bond lengths and angles at the carbene carbon atom, and in contrast to some data for imidazol-2-ylidenes ${ }^{51}$, triazolylidenes 15 appear to be the most stable heteroaromatic carbenes presently known.

It was established by $\mathrm{X}$-ray analysis that chelated anionocarbene complex 24 exists in a nonplanar conformation with respect to the fused Ni-O-arene ring [torsion angles $\varphi\left(\mathrm{C}^{1} \mathrm{~N}^{3} \mathrm{C}^{19} \mathrm{C}^{24}\right)$ 30.2,$\varphi\left(\mathrm{C}^{25} \mathrm{~N}^{6} \mathrm{C}^{13} \mathrm{C}^{48}\right) \quad 26.7^{\circ} ; \varphi\left(\mathrm{N}^{3} \mathrm{C}^{1} \mathrm{NiO}^{1}\right)-40.9^{\circ}, \varphi\left(\mathrm{N}^{6} \mathrm{C}^{25} \mathrm{NiO}^{2}\right)-41.6^{\circ}$ ] (Fig. 1). The adamantyl groups have a mutually cis-orientation. The non-planar structure can result from the direction of nickel hybrid orbitals. Furthermore, the distortion of the planar structure can be partially attributed to the steric hindrance due to the cis orientation of the bulky adamantyl groups.

Another interesting structural feature of complex $\mathbf{2 4}$ concerns the relatively small internal angles at the carbenoid nitrogen atoms $\left(103-104^{\circ}\right)$ (Fig. 2), which are closer to carbenic ones $\left(100^{\circ}\right)$, than to those of triazolium salts $\left(112^{\circ}{ }^{27 \mathrm{c}}\right)$. Furthermore, the bond lengths in the triazolium ring are reminiscent of those in triazolylidenes $\left(\mathrm{r} \mathrm{C}^{5}-\mathrm{N}^{1} 132-133 \mathrm{pm}\right.$ for complex 24 versus $134-135 \mathrm{pm}$ for carbene $15 \mathrm{~b}^{27 \mathrm{c}} ; \mathrm{r} \mathrm{C}^{5}-\mathrm{N}^{4} 137-138$ versus $138-139 \mathrm{pm} ; \mathrm{r} \mathrm{C}^{3}=\mathrm{N}^{2}$ $129-130$ versus $130 \mathrm{pm} ; \mathrm{r} \mathrm{C}^{3}-\mathrm{N}^{4} 138$ versus $138 \mathrm{pm}, \mathrm{r} \mathrm{N}^{1}-\mathrm{N}^{2} 139$ versus $\left.139 \mathrm{pm}\right)$. The $\mathrm{C}^{5}-\mathrm{Ni}$ bond length (approximately $189 \mathrm{pm}$ ) corresponds to that in analogous carbenic complexes of imidazol-2-ylidenes (e.g., with nickelecene $\mathrm{r} \mathrm{C}^{2}-\mathrm{Ni} 189-192 \mathrm{pm}^{52,53}$ ).

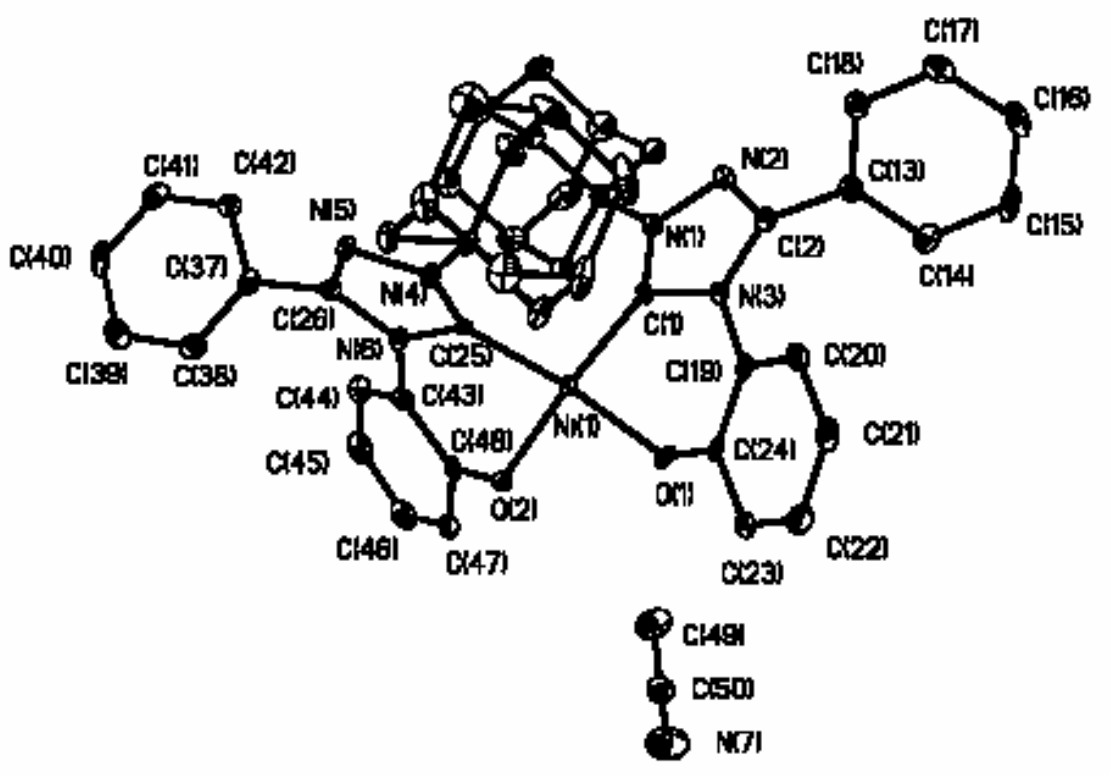

Figure 1. Structure of the acetonitrile solvate of complex $\mathbf{2 4}$ according to the X-ray diffraction study 


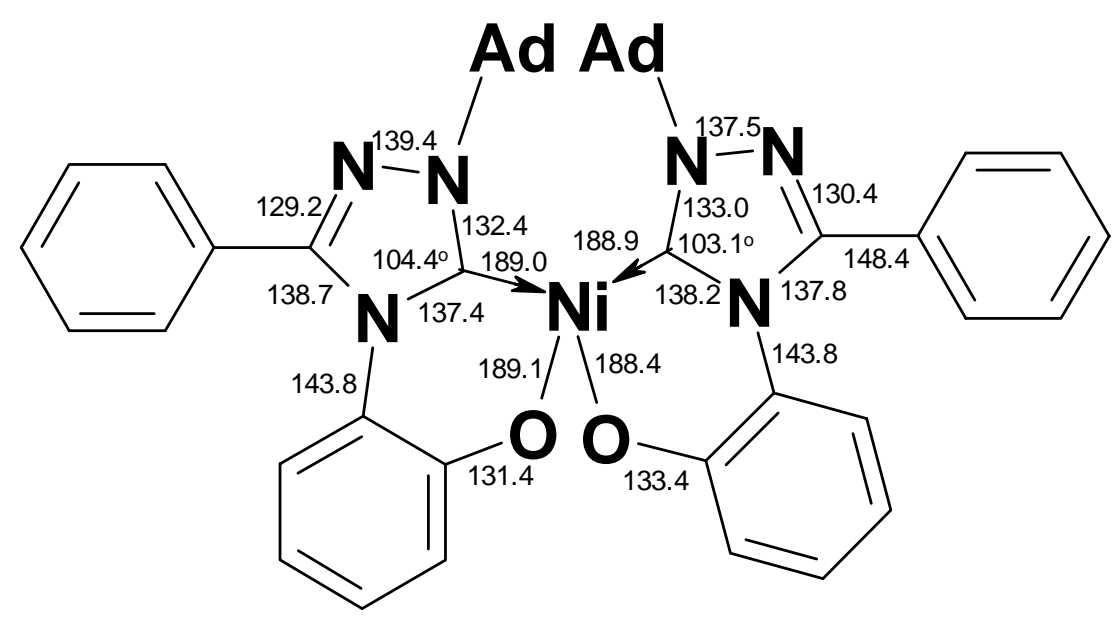

Figure 2. Bond lengths (pm) and the angle at the carbene carbon atom (degrees) in complex 24 according to the X-ray diffraction study

\section{Properties of heteroaromatic carbenes}

\subsection{Spectral properties}

The characteristic spectral features of compounds $\mathbf{1 5 a}-\mathbf{e}$ are the ${ }^{13} \mathrm{C}$ NMR signals for the carbene carbon atoms in the range $\delta 210-212$ ppm (c.f. the value of 214.6 ppm reported by Enders et al. ${ }^{8}$ for a triazolylidene). The ${ }^{1} \mathrm{H}$ NMR signals for the adamantyl and aromatic protons of carbenes 15a-c are shifted upfield compared with those for the corresponding salts $(\Delta \delta 0.2-0.5 \mathrm{ppm})$, and the absence of the $\mathrm{C}^{5} \mathrm{H}$ proton signal is due to the carbenic structure of these compounds. In the ${ }^{13} \mathrm{C}$ NMR spectrum of 19, the resonance of the $\mathrm{C}^{2}$ carbon is observed at $\delta 223.0 \mathrm{ppm}$. In the ${ }^{1} \mathrm{H}$ NMR spectrum of 19, a shift of all aromatic and adamantyl signals is also observed to be upfield compared with those of the corresponding salt signals $(\Delta \delta 0.4-0.6 \mathrm{ppm})$.

In the ${ }^{13} \mathrm{C}$ NMR spectra of the biscarbenes $20 \mathbf{a}-\mathbf{c}$, the carbene carbon signals appear at $\delta$ 208-228 ppm. It is interesting that those of the aromatic structure 20a appear appreciably downfield ( $\delta 228 \mathrm{ppm})$ with respect to the biscarbene with an aliphatic bridge, 20c $(\delta 208 \mathrm{ppm})$ and the monotriazol-5-ylidenes $(\delta 210 \mathrm{ppm})$, thus implying the presence of conjugation between carbene cycles and aromatic bridge in 20a. However, the same signal for $m$-substituted biscarbene 20b does not differ from that of the monocarbenic species, thus indicating a conjugation breach in the former compound. Furthermore, there is no essential difference in the position of the carbene carbon resonance for the $m$-phenylene- and $p$-phenylene-substituted biscarbenes 21a,b ( $\delta 228-229$ ppm).

In the ${ }^{1} \mathrm{H}$ NMR spectra of carbenes $\mathbf{1 5 , 2 0}$ it is also noteworthy that the location of the proton signals of the adamantyl methylene links nearest to nitrogen appear downfield (about $2.6 \mathrm{ppm}$ ). This cannot be attributed to salts $\mathbf{1 a - e , 8 a , b , 1 0 a , b}$, in which a cation causes an electron withdrawing effect (maximal value $\delta 2.24-2.29 \mathrm{ppm}$ ) and also to other signals of adamantyl 
protons for carbenes (the resonances of the carbene protons are shifted upfield in comparison with those of salts). Most likely, the adamantyl protons nearest to the nucleus experience deshielding due to their presence near the face of the carbene electron pair. In the case of the rigid structure of carbene 20a, these effects take place with both adamantyl groups while the more flexible structure of 20c permits the interaction of only one such group. For benzimidazol2-ylidene 19, a downfield shift of the signal is also observed ${ }^{27 a}$. However, this can also be attributed to deshielding of the fused aromatic ring (in the spectra of the corresponding benzimidazolium salt, the position of the related signal is similar). As expected, the other signals (aromatic and adamantyl protons) in the spectra of carbenes 20a-c,21a,b are shifted upfield in comparison with those of the corresponding salts.

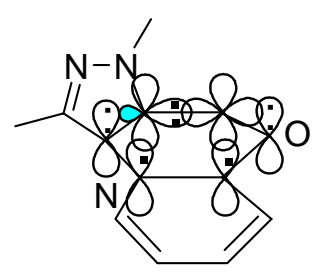

The most characteristic resonances in the ${ }^{13} \mathrm{C}$ NMR spectra of complexes $\mathbf{2 3 , 2 4}$ are those of the carbenoid carbon atoms $\mathrm{C}^{5}$ which range from $\delta 123-126 \mathrm{ppm}$. These values differ considerably from the carbenic values $(\delta 210-212 \mathrm{ppm})$ and fall in the region observed for azolium salts (cf., for salt $\mathbf{1 4} \delta 121.26 \mathrm{ppm}$, for azoline $\mathbf{1 6} 78.54 \mathrm{ppm}$ ). The difference between these values and those of carbenes ( $\Delta \delta$ about $90 \mathrm{ppm}$ ) can be considered to represent evidence in favor of aromaticity in the chelate rings of new complexes $\mathbf{2 3 , 2 4}$ (cf., for the recently synthesized carbenic lithium complexes, the chemical shifts for the carbenoid carbon differ only slightly from the carbenic ones in $\mathbf{2 3}(\Delta \delta 8-18 \mathrm{ppm}))^{13}$. For the known complexes of imidazol-2ylidene with nickelecene, the difference is larger $(\Delta \delta$ about $60-62 \mathrm{ppm}$, for monocarbenic complexes $\delta \mathrm{C}^{2} 159 \mathrm{ppm}$ and for biscarbenic ones is $\left.161 \mathrm{ppm}\right)^{52,53}$ but smaller than for new complexes $\mathbf{2 3 , 2 4}$.

\subsection{Basicity}

Stable nucleophilic carbenes are very strong bases (for 1,3-di-R-imidazol-2-ylidene, $\mathrm{R}=\mathrm{t}$-Bu, $i$ $\operatorname{Pr}$, the $\mathrm{p} K_{\mathrm{a}}$ is $22.7-24$ in dimethylsulphoxide- $\mathrm{d}_{6}$ depending on whether ion pairing is taken into account). In the context of neutral compounds, these values are inferior to only those of Schwesinger bases and other phosphazeneamines ${ }^{36,54}$. The basicity of benzimidazol-2-ylidene 19 is also very high $\left(\mathrm{p} K_{\mathrm{a}} 23.4\right.$ in benzene- $\mathrm{d}_{6}{ }^{55}$ measured according to the method described in reference ${ }^{36}$. However, this value can be compared only approximately with the above-mentioned data. 


\subsection{Insertion reactions into polar bonds}

As noted earlier, carbenes readily undergo reactions with acids and bases to form the products of carbene insertion into polar bonds $\mathrm{X}-\mathrm{H}$. The products of these reactions with $\mathrm{HC}$ are the corresponding azolines. Earlier, such products were obtained only by in situ reactions with highly electrophilic 1,2,4-triazolium and dithiolium salts ${ }^{8,56}$. In particular, it was proved that in the case of the reaction of 1,3,4-triphenyl-1,2,4-triazolium salt with sodium methylate in alcohol solution 5-methoxy-5H-1,2,4-triazoline was formed initially, but decomposed upon subsequent heating to the corresponding triazolylidene ${ }^{8}$. We have found that 2-tert-butoxyazoline 25 and 2 aminoazolines $\mathbf{2 6}$ are formed in situ from benzimidazolium salts and tert-butanol or secondary amines (morpholine, piperidine) in the presence of bases. The same products are formed in the reactions of isolable carbenes with the indicated reagents. Azolines represent the intermediate products in the syntheses of stable carbenes and are more or less easily converted to carbenes upon heating, dissolution, or removal of the excess proton-containing solvent ${ }^{56}$.<smiles>[X][R]N1c2ccccc2N([R])C1([R])N1CCCCC1</smiles>

$$
\mathbf{2 6}\left(\mathrm{X}=\mathrm{CH}_{2}, \mathrm{O} ; \mathrm{R}=\mathrm{Me}, \mathrm{Bn}\right) \quad \mathbf{D}
$$

The reactions of carbenes with water have been studied on the basis of in situ experiments for several years. At first it was thought that azolium hydroxides (hydroxyazolines) 27 were the initial products of such reactions (Scheme 7) because of their strongly basic reactions in aqueous solutions $^{57}$.

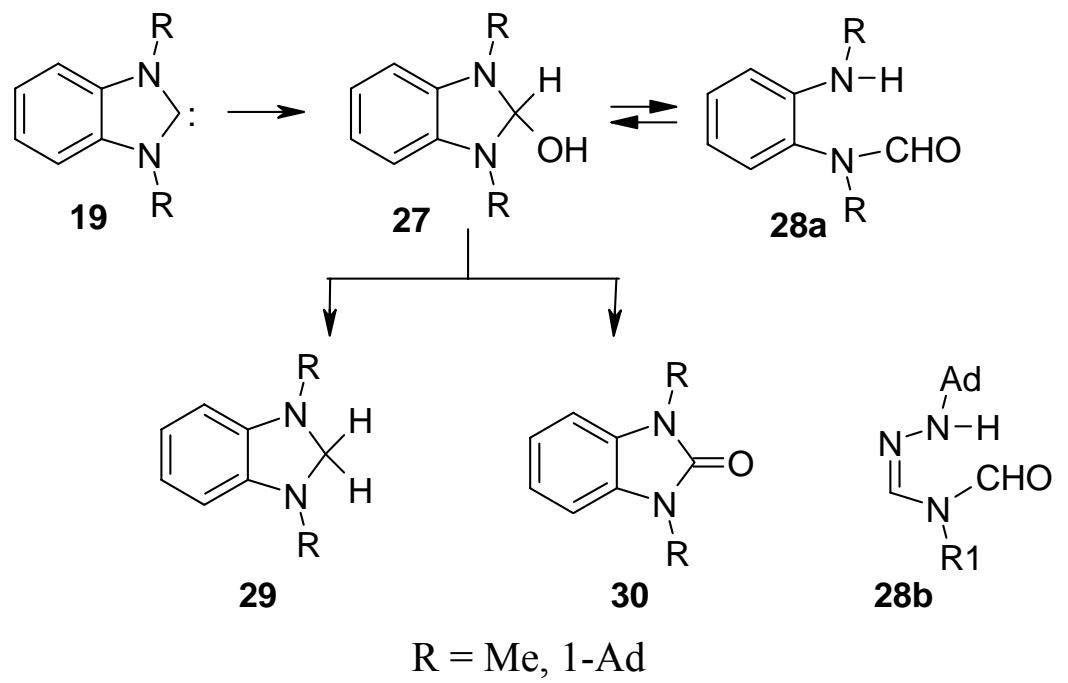

\section{Scheme 7}


Subsequently, on the basis of physical methods of analysis, the structure of $N$-formylamines 28, i.e. the products of the azolium ring opening of the intermediate 2-hydroxy- $2 \mathrm{H}$-azolines were confirmed ${ }^{58,59}$. However, in aqueous media, the ionic azolium hydroxides can be stabilized and characterized, and have been shown to be stable at room temperature for at least for one week ${ }^{60}$. It is also known that, when stabilized by electron withdrawing substituents (COOMe), 2hydroxybenzimidazolines can exist in a covalent form ${ }^{61}$.

The formation of $N$-formylamines $28 \mathbf{a}, \mathbf{b}$ by the reactions of stable carbenes $15 \mathbf{c}, 19$ with water was confirmed by us. However, if the substituents at the nitrogen atoms of the azolium ring are sterically hindered (hydrolysis of 1,3-di(1-adamantyl)benzimidazol-2-ylidene 19), azolium hydroxides 27 become sufficiently stable and their equilibrium with $N$-formylamine 28a can be detected by ${ }^{1} \mathrm{H}$ NMR spectroscopy even in solvents of low polarity such as chloroform-D.

The thermal dissociation of 2-hydroxyazolines $\mathbf{2 7}$ under vacuum to form a carbene and water proceeds only partially and disproportionation was observed to afford the appropriate azolones and azolines ${ }^{62}$. The formation of azolone in the reaction of 1,3-dimethyl-2-hydroxy- $2 \mathrm{H}$ benzimidazoline has also been observed earlier ${ }^{57}$, but the second product was considered to be 1-methylbenzimidazole. We found that heating in the presence of drying agents (barium or calcium oxides) does not change the outcome of the process, but in the presence of calcium hydride, $2 \mathrm{H}$-azolines are formed exclusively. The disproportionation of 2-hydroxyazolines probably proceeds by a carbenic or carbenoid mechanism (i.e., whether or not the reaction proceeds with participation of carbenes), and is connected with the intramolecular reduction of the intermediate benzimidazol-2-ylidenes by hydroxyazoline in a complex of type $\mathbf{D}^{63}$ similar to Arduengo's carbene H-complex ${ }^{64}$. In the presence of hydrides the reduction of the carbene takes place at the expense of the hydrogen eliminated during the interaction of the hydride with water evolved from the hydroxyazoline.

Taking into account the experimental facts, it is becoming clearer that another reduction process, related to the one described above, takes place upon heating of azolium formates ${ }^{65}$. Analogously to hydroxyazolines 27, azolium formates (2-formyloxyazolines) 4 can reduce the intermediate carbene, the product of dissociation of formyloxyazoline, to form $2 \mathrm{H}$-azoline $\mathbf{2 9}$ (Scheme 8). The sensitivity of the yield of the reaction to moisture confirms the carbene mechanism for this reaction. Meanwhile, the moisture-insensitive Leuckart reaction can proceed via the intramolecular reduction of the bond $\mathrm{C}=\mathrm{N}^{+}$. This pathway should become dominant in the absence of a $\mathrm{C}^{2} \mathrm{H}$-proton in the structure of salt. 


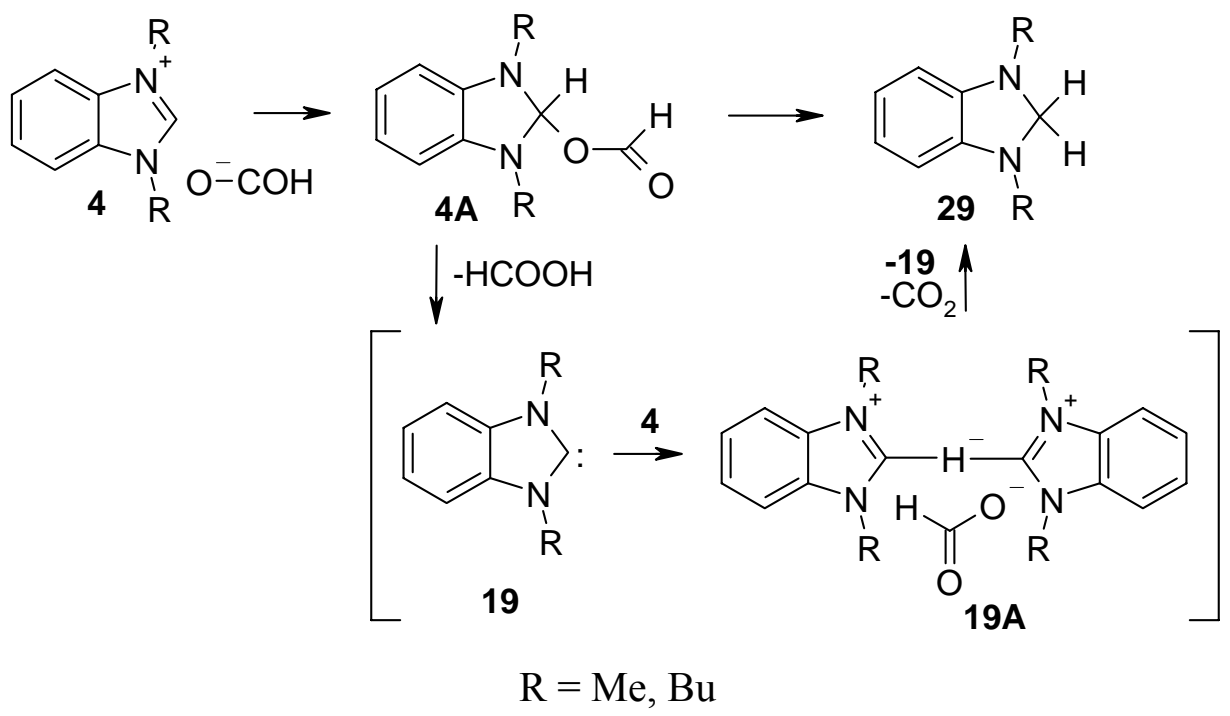

\section{Scheme 8}

\subsection{C-H Insertions}

As mentioned above, the carbenes 15,19 exhibit a different reactivity toward acetonitrile. Earlier, similar outcomes have been described for the reactions of carbenes with $\mathrm{C}-\mathrm{H}$ acids such as acetonitrile to form the corresponding substituted alkanes ${ }^{2,66}$. The reactions of electrophilic carbenes with acetonitrile have been studied by kinetic methods ${ }^{67}$. The insertion of the nucleophilic 1,3-diphenylimidazolin-2-ylidene in situ (generated from its dimer) into acidic C-H bonds of acetonitrile was described initially by Wanzlick et al. ${ }^{68}$, who used a high temperature process $\left(150-180^{\circ} \mathrm{C}\right)$. In situ transformations of $\mathrm{HC}$ dithiolylidenes with malonic nitrile $\left(\mathrm{p} K_{\mathrm{a}} 11\right.$ 12) and cyclopentanone $\left(\mathrm{p} K_{\mathrm{a}} 15\right)^{2,56}$ are also known. In the articles cited in reference ${ }^{56}$, the insertion of $\mathrm{HC}$ dithiol-2-ylidene is considered to result from the formation of the intermediate ylide complexes.

The transformations of isolable nucleophilic $\mathrm{HC}$ with acetonitrile have not been studied until recently. Herrmann and co-workers ${ }^{14,15,22}$ noted that imidazol-2-ylidenes do not react with acetonitrile, and, in fact, an acetonitrile-tetrahydrofuran mixed solvent was used for the preparation of metal-carbene complexes. Moreover, Enders et al. ${ }^{12}$ expressed the opinion that the products of $\mathrm{C}-\mathrm{H}$ insertion could be formed only beyond the limits of thermal stability (more than $\left.150{ }^{\circ} \mathrm{C}\right){ }^{12}$. In a more recent article by Arduengo et al. ${ }^{35}, \mathrm{C}-\mathrm{H}$ insertion reactions of mainly the saturated carbene, 1,3-dimesitylimidazolin-2-ylidene, were described (including the reaction with acetonitrile). However, the crystalline structures of the products were not determined.

On the basis of the reactions of 1,2,4-triazol-5-ylidenes 15, benzimidazol-2-ylidenes 19 and imidazol-2-ylidene, we have demonstrated that the $\mathrm{C}-\mathrm{H}$ insertion reaction is one of the most important chemical properties of HC. Since the basicity of these carbenes is perceptibly lower than that for cyanomethanide-ion, these reactions probably proceed by a concerted mechanism. However, under conditions of increasing of $\mathrm{C}-\mathrm{H}$ acidity, e.g., by changing to 1,3dimethylbarbituric acid ( $\mathrm{p} K_{\mathrm{a}} 4.68$ in water) the direction of the triazolylidene reaction changes: 
carbene 15e reacts with a complete elimination of the $\mathrm{C}^{5} \mathrm{H}$ proton to form triazolium barbiturate 31, i.e. the insertion into $\mathrm{C}-\mathrm{H}$ bond does not occur ${ }^{55}$. It should be noted that the related 2nitromethyl-1,3-dibenzyl-2 $\mathrm{H}$-benzimdazoline, which is a product of insertion into the $\mathrm{C}-\mathrm{H}$ bond of a weaker acid, nitromethane $\left(\mathrm{pK}_{\mathrm{a}}\right.$ 10.2-10.6) was naturally produced as a covalent compound

${ }^{69}$, although its partial dissociation leads to oxidative transformation to the corresponding azolium nitrate. The chemical shift of the $\mathrm{CH}$ proton of the barbiturate cycle 31 appears at $\delta 4.05$ ppm and the $\mathrm{C}^{5}$ atom evidences a ${ }^{13} \mathrm{C}$ resonance at $\delta 77.11 \mathrm{ppm}$ that presumably corresponds to the $\mathrm{C}$-anionic structure. The ${ }^{13} \mathrm{C}$ chemical shift for the $\mathrm{C}^{5} \mathrm{H}$ resonance group of triazolium cycle (10.47 ppm) implies the absence of hydrogen bonding between the triazolium and barbiturate rings.

\subsection{Reactions with chalcogens}

Sulfur is considered to be the best trap for carbenes ${ }^{66 a}$. We therefore studied the reactions of $\mathbf{1 5 a}$ and 19 with sulfur as well as the reaction of $\mathbf{1 5 c}$ with selenium. Each of these carbenes reacts very rapidly (in a few minutes) to form thiones 32a, 33a and selenone 32b, respectively. In the case of the reaction of carbene 19 with sulfur, a deeply-colored (red and brown) compound $\mathbf{3 4}$ was also isolated. Compound 34 has the composition $\left(\mathrm{C}_{27} \mathrm{H}_{34} \mathrm{~N}_{2}\right)_{2} \mathrm{~S}_{7} \cdot 4 \mathrm{C}_{6} \mathrm{H}_{6}$ and includes benzene of solvation [probably the structure of the compound is of type $\mathrm{Crb}^{+}-\mathrm{S}^{-}\left(\mathrm{S}^{+}=\mathrm{S}^{-}\right)_{3}=\mathrm{Crb}$, where $\mathrm{Crb}$ is a fragment of carbene 19 in an azolium state $\left(\mathrm{Crb}^{+}-\right)$or in a neutral form $\left.(\mathrm{Crb}=)\right)$. Compound 34 is very unstable to oxygen and moisture, and upon dissolution in chloroform it forms thione 33a in a $16 \%$ yield and salt $4 \mathbf{c}$ with the anion $\mathrm{HS}_{3} \mathrm{O}_{4}{ }^{-}$in $60 \%$ yield. It has not yet proved possible to record NMR or IR spectra for $\mathbf{3 4}$, nor has it been possible to determine the X-ray crystal structure due to its facile decomposition in polar solvents. The major reason why the substance was isolated is its low solubility in benzene, from which it is precipitated. On the basis of the composition, properties and the above-mentioned features of chemical behavior, compound $\mathbf{3 4}$ can probably be considered to be a hypervalent compound of sulfur. The yields of thione 33a differ considerably if an excess of the carbene (49\%) or an excess of sulfur (90\%) is employed. We believe that the formation of hypervalent compound $\mathbf{3 4}$ is responsible for these variations in yields.

Treatment of biscarbene 20a with sulfur in toluene solution produced thione 35 in $51 \%$ yield; however, the formation of the latter was accompanied by a series of side products that are presumed to be hypervalent compounds on the basis of TLC.

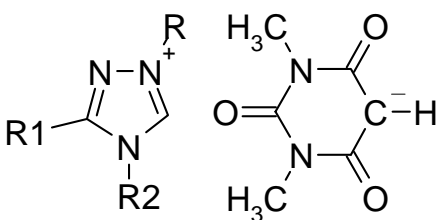

31

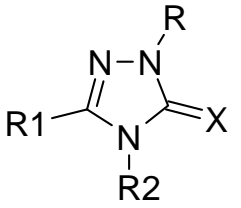

32a,b<smiles>[R]n1c(=S)n([R])c2ccccc21</smiles>

33a,b

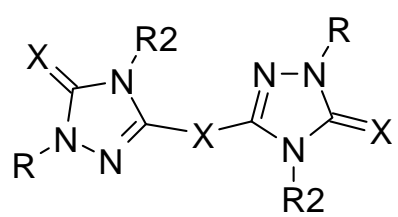

35

$31 \mathrm{R}=\mathrm{t}-\mathrm{Bu} ; \mathrm{R}_{1}=\mathrm{R}_{2}=\mathrm{C}_{6} \mathrm{H}_{5} ;$ 32a,b,33a,35 $\mathrm{R}=1$-Ad; 32a $\mathrm{R}_{1}=\mathrm{R}_{2}=\mathrm{C}_{6} \mathrm{H}_{5} ;$ 32b $\mathrm{R}_{1}=\mathrm{R}_{2}=\mathrm{C}_{6} \mathrm{H}_{4} \mathrm{Br}-p$; 33b $\mathrm{R}=\mathrm{Bn} ; 35 \mathrm{R}_{2}=\mathrm{C}_{6} \mathrm{H}_{5}, \mathrm{X}=\mathrm{C}_{6} \mathrm{H}_{4}-p$ 
Complex 23 exhibits enhanced chemical stability in comparison with related carbenes. Nevertheless, this compound undergoes hydrolysis in acetonitrile solution and other solvents in the presence of moisture. Complex 23 reacts readily with sulfur in acetonitrile solution (for $0.5 \mathrm{~h}$ at room temperature) to yield thione 36. Treated with sulfur and epichlorohydrin, oxidation of $\mathbf{2 3}$ occurs to produce triazolone 37 . The ouctome of this reaction (but not the reaction with two nucleophilic centers, which was assumed at first) is possibly linked to the high rate of the epichlorohydrin reaction with the sulfur atom of thione 36, followed by the rapid processes outlined in Scheme 9.

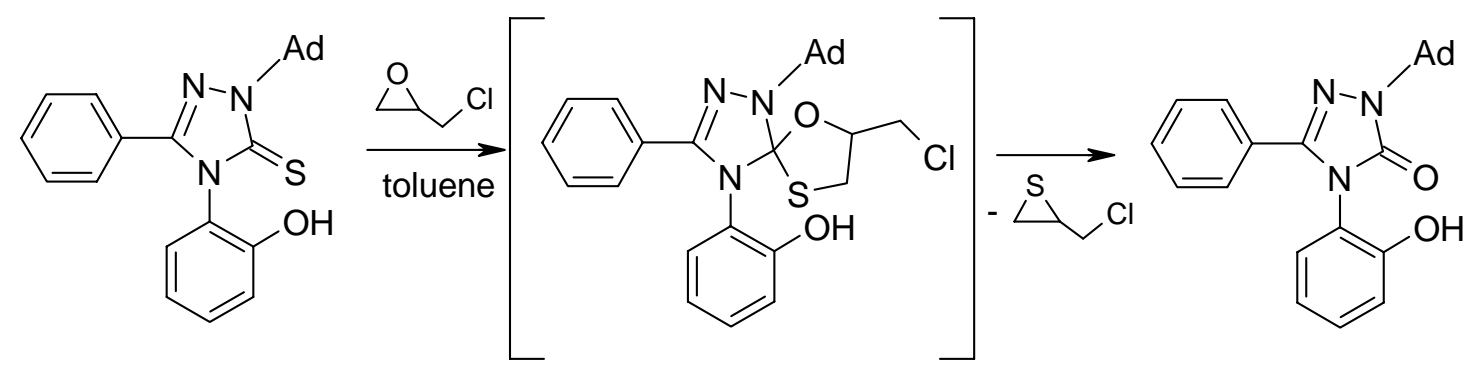

36

37

\section{Scheme 9}

The reaction conversion of $\mathbf{3 6}$ to $\mathbf{3 7}$ represents a new modification of the Dachlauer-Jackel reaction of oxiranes with thiocarbamides ${ }^{70}$. In contrast to the known reactions with $\mathrm{NH}-$ functionalized thiocarbamides, the reaction with the $N$-substituted derivative of thiocarbamide 36 proceeds very readily (even in low polarity toluene). Thus, with the help of previous quaternization and thione formation, the reaction can be used for the synthesis of complicated azolone derivatives of type $\mathbf{3 7}$ and of thiiranes from azoles.

\subsection{Complex formation}

It is now clear that the formation of hypervalent compounds with the atoms of elements containing vacant $s, p, d$ or $f$-orbitals is a characteristic chemical property of stable nucleophilic carbenes $22,23,69$. This interaction proceeds particularly easily with transition elements, especially the heavier metals.

Given the absence of literature data on biscarbene complexes with conjugated intercarbene bonds we decided to attempt the synthesis of the first stable bimetallic complexes of biscarbene 20a with a rare earth element, particularly with the praseodymium (III) ion. Treatment of praseodymium trisacetylacetonate or tris(dibenzoylmethanide) with biscarbene 20a in toluene solution in a 2:1 molar ratio resulted in the formation of bimetallic complexes of composition $\mathrm{R}_{3} \operatorname{PrCrb}-\mathrm{X}-\mathrm{CrbPrR} \mathrm{P}_{3}$ ( $\mathrm{R}$ represents an acetylacetonate or dibenzoylmethanide fragment; $\mathrm{Crb}$ and $X$ are a biscarbene link 20a). These complexes exhibit high solubility in polar aprotic and aromatic solvents and low solubility in saturated hydrocarbons. 
Over the past ten years, many carbene reactions with electrophilic compounds of the nonmetals (activated with electron-withdrawing substituents) have been described. The list includes such compounds as thionyl chloride, sulfur (II) chloride, pentafluoroiodobenzene, molecular halogens (iodine, chlorine), phenylphosphortetrafluoride and phenylphosphinidene ${ }^{23,71}$. As noted above, a hypervalent compound of molecular sulfur can also be formed. Moreover, the fact that activated sulfides and disulfides also react with $\mathrm{HC}$ was shown by the transformation of carbenes 19 and 15b,c with bis(p-nitrophenyl)sulfide and disulfide ${ }^{27 d, 64}$. In these reactions, deeply colored (from red and violet to red and orange) and hydrolytically sensitive-hypervalent compounds of sulfur $38\left[\mathrm{X}=\mathrm{S}\left(\mathrm{C}_{6} \mathrm{H}_{4} \mathrm{NO}_{2}\right)_{2} ; \mathrm{S}_{2}\left(\mathrm{C}_{6} \mathrm{H}_{4} \mathrm{NO}_{2}\right)_{2}\right]$ are formed (for the complex of carbene 19 with sulfide, the absorption band appears at $\lambda 490 \mathrm{~nm}$ ). The absence of carbene interaction with the related 4.4'-dinitrodiphenyloxide suggests that the C-S bond in $\mathbf{3 8}$ is formed at the expense of the $s p^{2}-d$ interaction.

The products of the HC reaction with sulfur can be used for the synthesis of various heterocyclic compounds. Thus, the thionation of carbenes was used to produce thiirane derivatives of azolones ${ }^{72}$. Correspondingly, $\mathrm{N}$-thiiranomethyl derivatives $\mathbf{4 0 a}-\mathbf{d}$ are formed by the interaction of azoles 39a-d (imidazoles, benzimidazoles, benzothiazole) with epichlorohydrin and sulfur via the intermediate $N$-(2,3-epoxypropyl)azolium salts 41a-c, appropriate $N$-glycidylazolthiones, and zwitter-ions $\mathbf{4 2}$.
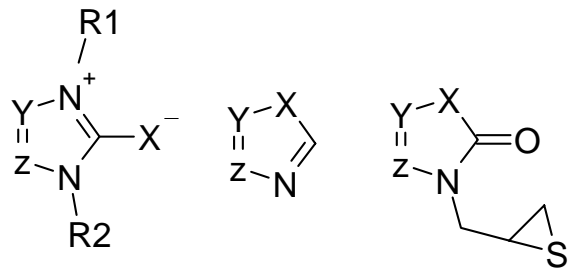

$38 \mathbf{a}-\mathbf{d}$

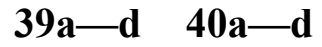

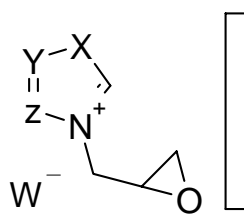

$41 \mathrm{a}-\mathrm{c}$

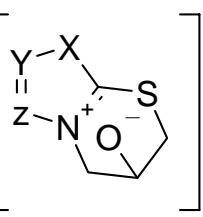

42

38a,c $X=\mathrm{S}\left(\mathrm{C}_{6} \mathrm{H}_{4} \mathrm{NO}_{2}\right)_{2}, \mathbf{3 8 b}, \mathbf{d ~} \mathrm{S}_{2}\left(\mathrm{C}_{6} \mathrm{H}_{4} \mathrm{NO}_{2}\right)_{2}$; 38a,b $\mathrm{Y}, \mathrm{Z}=(\mathrm{CH})_{4}, \mathrm{R}_{1}=\mathrm{R}_{2}=1$-Ad; 38c,d $\mathrm{Y}=\mathrm{N}$, $\mathrm{Z}=\mathrm{CC}_{6} \mathrm{H}_{5} ; \mathrm{R}_{1}=\mathrm{R}_{2}=\mathrm{C}_{6} \mathrm{H}_{4} \mathrm{Br}-p ; \mathbf{3 9}-\mathbf{4 1}, \mathbf{4 2} \cdot \mathbf{H C l O}_{4}$ a X $=\mathrm{NCH}_{3}, \mathrm{Y}, \mathrm{Z}=(\mathrm{CH})_{4} ; \mathbf{b} \mathrm{X}=\mathrm{NCH}_{3}, \mathrm{Y}=$ $\mathrm{Z}=\mathrm{CH} ; \mathbf{c} \mathrm{X}=\mathrm{NCH}_{3}, \mathrm{Y}=\mathrm{Z}=\mathrm{CC}_{6} \mathrm{H}_{5} ; \mathbf{3 9}, \mathbf{4 0} \mathbf{d} \mathrm{X}=\mathrm{S} ; \mathrm{Y}, \mathrm{Z}=(\mathrm{CH})_{4}$

The carbenoid reaction of $N$-allylbenzimidazolium salts 43 was used to produce thiiranomethylazolones $\mathbf{4 0}$ by the thionation of salts in the presence of bases, halogencyclization of the intermediate thiones 44, and a ring transformation of the formed 2halogenmethylazolothiazolium 45 and 3-halogenazolo-1,3-thiazinium salts $\mathbf{4 6}$ under the action of alkalis $^{73}$. 


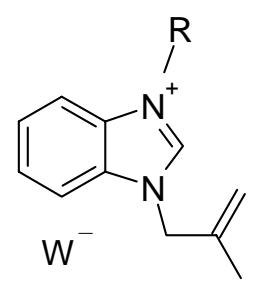

43

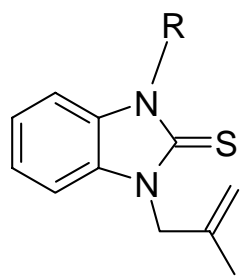

44

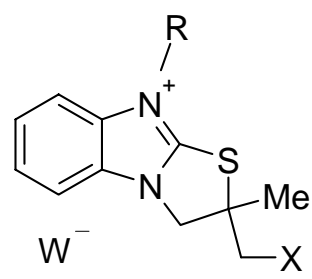

45

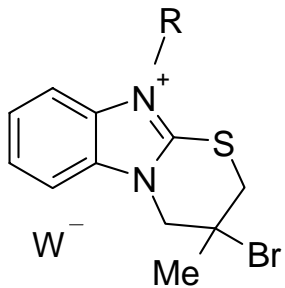

46

43,44 a $\mathrm{R}=\mathrm{CH}_{3} ; \mathbf{b ~} \mathrm{CH}_{2} \mathrm{C}_{6} \mathrm{H}_{5} ; \mathbf{4 5 , 4 6}$ a $\mathrm{X}=\mathrm{Br}, \mathrm{R}=\mathrm{CH}_{3}, \mathbf{b} \mathrm{X}=\mathrm{I}, \mathrm{R}=\mathrm{CH}_{3} ; \mathbf{4 3 , 4 5 , 4 6} \mathrm{W}=\mathrm{ClO}_{4}$, $\mathrm{Br}, \mathrm{I}$

\subsection{Reactions of heteroaromatic carbenes with double bonds}

The alkylation of HC by compounds with activated multiple bonds proceeds in an unusual fashion. The azolium structure that is characteristic of the reaction products of carbenes with alkylhalogenides is not formed, because in this case a cycloaddition takes place, followed by transformation of the initial adduct by a proton migration. Such a reaction pathway was observed by Jones and co-workers ${ }^{74}$ for the first time in the reaction of cyclopropenylidene with maleic esters.

Enders et al. ${ }^{8}$ carried out variations of this type of reaction by treating the stable triazolylidene $15\left(\mathrm{R}=\mathrm{R}_{1}=\mathrm{R}_{2}=\mathrm{C}_{6} \mathrm{H}_{5}\right)$ with maleic and fumaric esters $(\mathrm{X}=\mathrm{Y}=\mathrm{COOR})$, acrylonitrile $(X=H, Y=C N)$, nitrostyrene $\left(X=H, Y=N_{2}\right)$, and maleimide $(X, Y=-$ CONRCO-, $\mathrm{R}=\mathrm{Me}, \mathrm{Bu}, \mathrm{Ph}$, Scheme 10). These authors expressed the opinion that initiallyformed carbanion 46 is in equilibrium with spirocyclopropane 47. In carbanion 46, a proton shift $(1,2-\mathrm{H})$ takes place with the formation of the corresponding azolylidenesuccinimide 48 . The presence of a carbanion in the chain of reactions is confirmed by the formation of the oligomeric addition product, which results from the nucleophilic attack of a carbanion by the second molecule of olefin.

In the work described in reference ${ }^{63}$ the reactions of two carbene classes, benzimidazolylidene 19 and triazolylidene 15c, with $N$-phenylmaleimide are described. It turned out that 19 gives mainly oligomeric products and that $15 \mathrm{c}$ forms the product of addition and proton shift, 48. The reason for such a difference relates to a large contribution of cyclopropane structure $\mathbf{4 7}$ in the equilibrium $\mathbf{4 6} \rightleftarrows \mathbf{4 7}$ for triazolylidene, due to the greater electron deficiency of the triazolium cycle compared to that of benzimidazolium. A decrease in the concentration of carbanion 46 and maleimide in the mixture due to the formation of 47 leads to disruption of chain growth, and, as a result, transformation of anion 46 into olefin 48. 


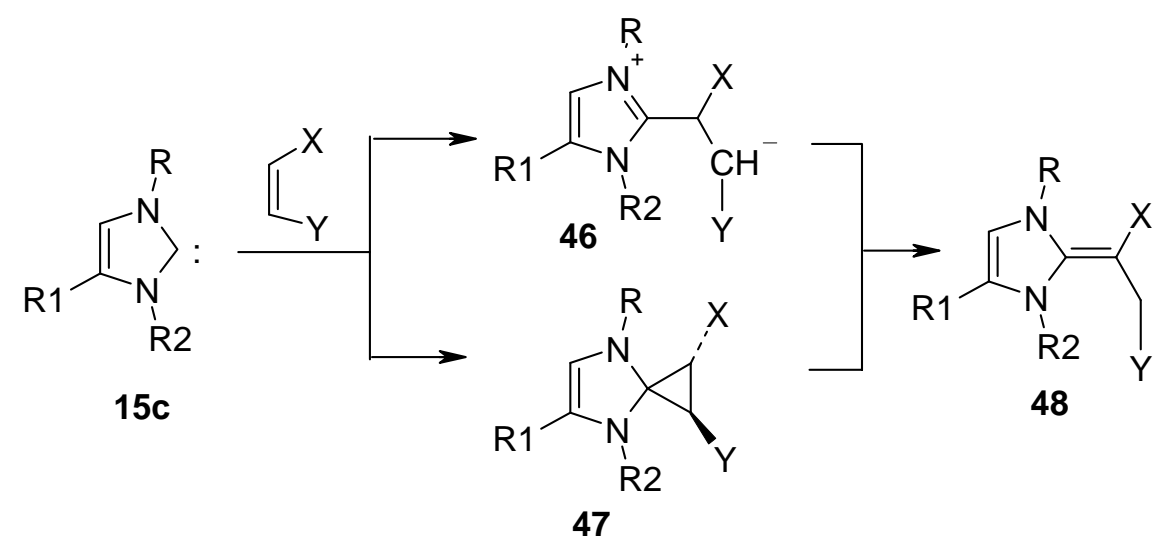

$48 \mathrm{R}=1-\mathrm{Ad} ; \mathrm{R}_{1}=\mathrm{R}_{2}=\mathrm{C}_{6} \mathrm{H}_{4} \mathrm{Br}-p ; \mathrm{X}, \mathrm{Y}=-\mathrm{CON}(\mathrm{Ph}) \mathrm{CO}-$

\section{Scheme 10}

The possibility of the interaction of carbenes 15,19 with carbon dioxide was considered in the work described in the reference ${ }^{63}$. In the absence of moisture the reaction does not proceed; however, in the presence of water, azolium hydrocarbonates 49 are formed almost instantly and in quantitative yield. The reason why benzimidazol-2-ylidene $\mathbf{1 9}$ does not react is possibly due to steric hindrance of the approach of the reagent to the carbene centre.<smiles>[R][Y4]1c([R])[n+]([R])cn1[R2]</smiles>

49<smiles>[R2]n1cc[n+]([R])c1C(=O)[O-]</smiles>

50<smiles>[R2]n1cc[n+]([R])c1C(=O)c1ccccc1C(=O)[O-]</smiles>

51<smiles>[R][Y]1c([R1])c(CC2CO2)n1[R2]</smiles>

52

49,51,52 $\mathrm{R}=1-\mathrm{Ad} ; \mathrm{R}_{1}=\mathrm{R}_{2}=\mathrm{C}_{6} \mathrm{H}_{4} \mathrm{Br}-p$

However, in the case of more basic and less sterically-hindered carbenes, i.e. 1,3diisopropylimidazol-2-ylidene, this reaction turned out to be possible. As a result, heterocyclic zwitter-ion of type $\mathbf{5 0}$ was isolated ${ }^{75}$. Perhaps such transformations are also possible for hydrogenated analogues of imidazol-2-ylidene and acyclic carbenes.

More active phthalic anhydride reacts with triazolylidene $15 \mathrm{c}$ very easily to form acyltriazolium zwitter-ion $\mathbf{5 1}{ }^{63}$. The latter compound is characterized by high ability to moisture and is probably analogous to $N$-acylpyridinium salts.

The reactions with organic oxides are particularly interesting from the point of view of obtaining spiro-heterocyclic compounds. They also open new possibilities for the production of 
small ring derivatives with heterocyclic ion fragments if the property of small ring-to-ring transformation is used in a similar fashion to the one described in reference ${ }^{76}$. In particular, the reaction of carbenes $\mathbf{1 5 b , c}$ with epichlorohydrin in the presence of sodium perchlorate produced C-glycidylazolium salts for the first time as shown by the example of 3-(2,3-epoxypropyl)derivatives, 52. The side product in this interaction is the 5-unsubstituted triazolium salt which is formed in $10-30 \%$. The characteristic feature of the ${ }^{1} \mathrm{H}$ NMR spectra of salts $\mathbf{5 2}$ is the presence of a proton signal for a $\mathrm{CH}_{2} \mathrm{C}$ moiety with azolium nucleus shifted upfield $(2,8 \mathrm{ppm})$ compared with the signals for the $\mathrm{CH}_{2} \mathrm{~N}$ moieties of known $N$-glycidylazolium salts $(4,0-4,9 \mathrm{ppm})^{77}$. Most likely, the reason for such a shift is not only low acceptor properties of triazolium cation in the 5 position compared to the nitrogen atom $\mathrm{N}^{+}$, but also the detected shielding of these protons from the side of the $\mathrm{C}-\mathrm{H} \sigma$-bonds of the adamantyl group.

\subsection{Reactions of cryptocarbenes}

Except when pure carbenes are used as reagents in organic synthesis, carbene generation in situ has often been used for the study of carbene reactions. This approach is well known since pure carbenes have often proved inaccessible. This approach is broadly used in the chemistry of triplet carbenes ${ }^{24}$, and became popular following the work of Bertrand et al. ${ }^{23}$ on the synthesis of phosphanylcarbenes, via photolysis of diazo precursors. Another potential application of carbenic and related reactions is for the preparation of the compounds, which in some conditions can take part in carbenoid processes. Even earlier, Wanzlick et al. employed carbene dimer reactions, particularly bisbenzothiazol-2-ylidenes and bisimidazolin-2-ylidenes ${ }^{2}$. These species can also be considered to be cryptocarbenes (latent carbenes), though the possibility of the carbene dimer dissociation is still being discussed ${ }^{78}$.

We found that the properties of cryptocarbenes are also useful in the context of cyanomethyl derivatives of azolines which are capable of reaction with piperidine and nitromethane to afford the carbene insertion products into $\mathrm{C}-\mathrm{H}$ and $\mathrm{N}-\mathrm{H}$ bonds $\mathbf{5 3 , 5 4}{ }^{69}$ (Scheme 11). 


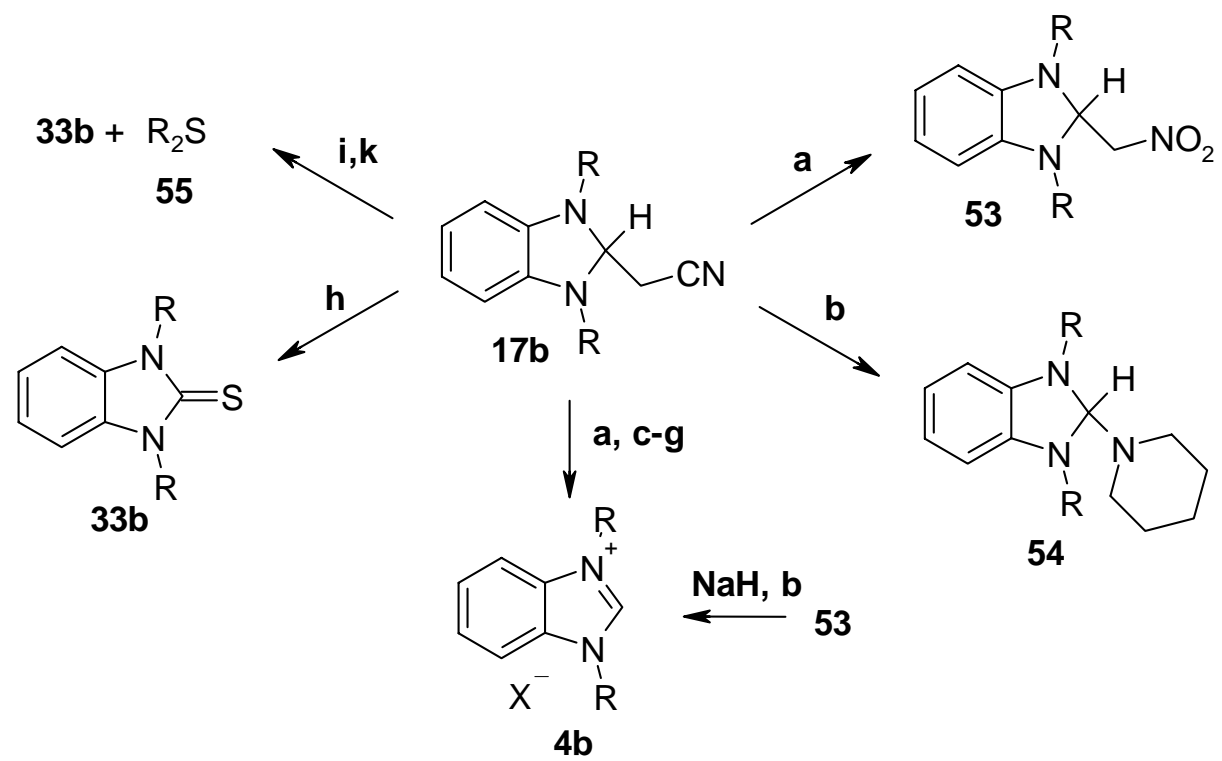

Reagents: a $\mathrm{CH}_{3} \mathrm{NO}_{2} ;$ b $\mathrm{HN}\left(\mathrm{CH}_{2}\right)_{5} ;$ c $\mathrm{HCl}, \mathrm{HClO}_{4}, \mathrm{CF}_{3} \mathrm{COOH}, \mathrm{AcOH} ;$ d $\left(\mathrm{CH}_{3}\right)_{2} \mathrm{SO}_{4} ;$ e epichlorohydrin; f methyliodide; $\mathbf{g} \quad \mathrm{BrC}_{6} \mathrm{H}_{3}\left(\mathrm{NO}_{2}\right)_{2}-2,4 ; \mathbf{h} \quad \mathrm{S} ; \quad \mathbf{i} \quad\left(\mathrm{O}_{2} \mathrm{NC}_{6} \mathrm{H}_{4} \mathrm{~S}\right)_{2} ; \mathbf{k} \quad \mathrm{R}_{2}^{1} \mathrm{~S}_{2}$. 4b,17b,33b,53,54 R = $\mathrm{CH}_{2} \mathrm{Ph} ; 55 \mathrm{R}^{1}=\mathrm{O}_{2} \mathrm{NC}_{6} \mathrm{H}_{4}$.

\section{Scheme 11}

With alkylating reagents azolium salts 4 were produced, while in the case of methyliodide an intramolecular cleavage of the intermediate azolinium salt 17A occurs simultaneously and leads to the formation of unsymmetrical azolium cation $4 e^{69}$ (Scheme 12). Cyanomethyl group performs the role of a base in the cleavage by its anionoid elimination. The reaction of azoline $\mathbf{1 7} \mathbf{b}$ with sulfur leads to the corresponding thione $\mathbf{3 3 b}$ and with disulfides the desulfurization reaction results in the formation of sulfides 55 (Scheme 11). Simultaneously with dibenzothiazolyldisulfide a reduction of disulfide to thione $\mathbf{3 3 \mathbf { b }}$ was observed. All the foregoing reactions are probably of the carbenoid type.

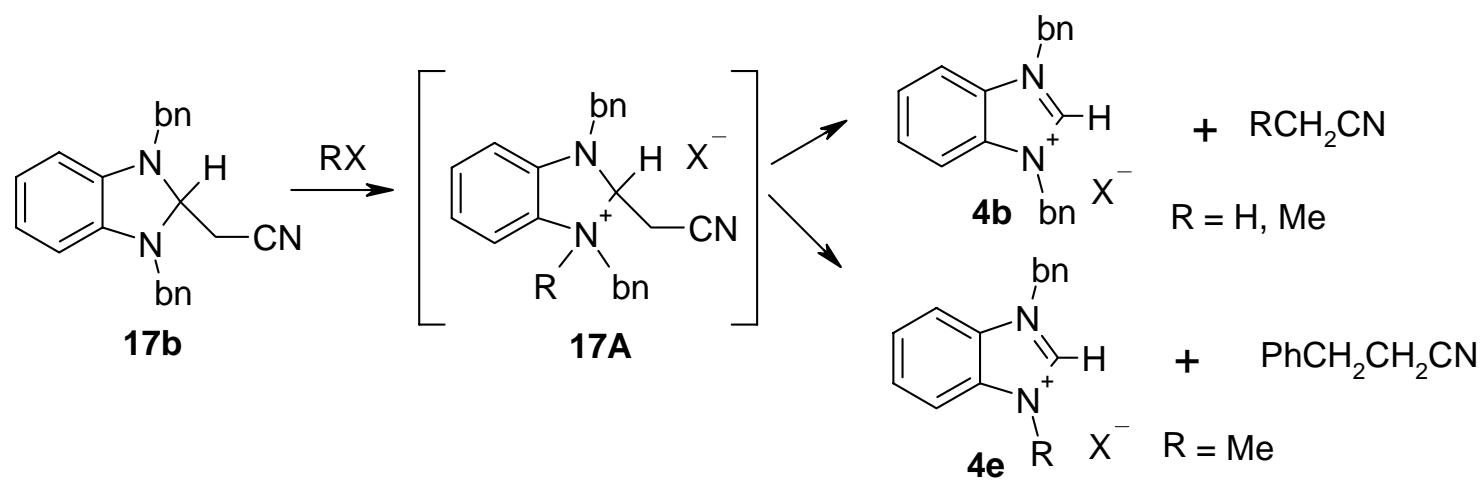

Scheme 12 
When treated with metal compounds the related process takes place and results in carbenic metal complexes. For example, the reaction of 1,3-dimethyl-2-cyanomethyl-2H-benzimidazoline with $\mathrm{CuI}$ and $\mathrm{NiI}_{2}$ results in biscarbenic and triscarbenic complexes of copper $\left(\mathrm{CuCrb}_{2} \mathrm{I}\right)$ and nickel $\left(\mathrm{NiCrb}_{3} \mathrm{I}_{2}\right)$. Previously, nickel complexes of this type had proved to be inaccessible. The copper complex is analogous to that obtained from imidazol-2-ylidenes ${ }^{79}$. At the same time it is worth stressing that the reactions of azolines as cryptocarbenes proceed appreciably slower than those of carbenes.

\section{Conclusions}

In conclusion, it should be noted that only the results from the HC chemistry that are relevant to the particular interests of the authors have been included in the present review. It was not our objective to develop an exhaustive analysis of the literature in this rapidly developing field of organic chemistry. In particular, this relates to the chemistry of carbene precursors where in the 1,2,4-triazole series a considerable contribution was made by Enders and co-workers (see, e.g. ${ }^{78}$ ). Nevertheless, even with the results presented here, it is clear that the isolation of pure carbenes presents many new and exciting possibilities for studying the transformations of these relatively new reagents that had only been available earlier on an in situ basis. Further development of $\mathrm{HC}$ chemistry should make these substances significantly accessible to permit their widespread use in organic synthesis and catalysis. Other intriguing possibilities include the generation of carbenes with the help of special reagents and the imitation of carbenes using more stable and convenient compounds (cryptocarbenes).

\section{Acknowledgements}

We thank the Ukrainian Foundation of the Fundamental Research and Education and Science Ministry of Ukraine for financial support (Grant No. 03.07/00). We also thank the Robert A. Welch Foundation for financial support of the X-ray diffraction studies.

\section{References}

1. (a) Breslow, R. J. Am. Chem. Soc. 1957, 79, 1762. (b) Breslow. R. J. Am. Chem. Soc. 1958, 80, 3719.

2. For a review, see Shvaika, O. P.; Korotkikh, N. I.; Aslanov, A. F. Chemistry of Heterocyclic Compounds (Latvia) 1992, 9, 1155.

3. Igau, A.; Grutzmacher, H.; Baceiredo, A.; Bertrand, G. J. Am. Chem. Soc. 1988, 110, 6463.

4. Gillette, G. R.; Baceiredo, A.; Bertrand, G. Angew. Chem. Int. Ed. 1990, 102, 1486. 
5. Arduengo, A. J.; Harlow, R. L.; Kline, M. J. Am. Chem. Soc. 1991, 113, 361.

6. Arduengo, A. J.; Dias, H. V. R.; Harlow, R. L.; Kline, M. J. Am. Chem. Soc. 1992, 114, 5530 .

7. Alder, R. W.; Allen, P. P.; Murray, M.; Orpen, A. G. Angew. Chem. Int. Ed. 1996, 35, 1121.

8. Enders, D.; Breuer, K.; Raabe, G.; Runsink, J.; Teles, J. H.; Melder, J.-P.; Ebel, K.; Brode S. Angew. Chem. Int. Ed. 1995, 34, 1021.

9. Arduengo, A. J.; Goerlich, J. R.; Marshall, W. J. Liebigs Ann./ Recueil. 1997, 365.

10. Wanzlick, H. W. Angew. Chem. 1962, 74, 129.

11. Kuhn, N.; Kratz, T. Synthesis 1993, 12, 561.

12. Enders, D.; Breuer, K.; Runsink, J.; Teles, J. H. Liebigs Ann. Chem. 1996, 2019.

13. Alder, R. W.; Blake, M. E.; Bortolotti, C.; Bufali, S.; Butts, C. P.; Linehan, E.; Oliva, J. M.; Orpen, A. G.; Quayle, M. A. J. Chem. Soc., Chem. Commun. 1999, 3, 241.

14. Herrmann, W. A.; Elison, M.; Fischer, J.; Kocher, K.; Artus, G. R. J. Chem. Eur. J. 1996, 2, 1627.

15. Herrmann, W. A.; Elison, M.; Fischer, J.; Kocher, K.; Artus, G. R. J. Chem. Eur. J. 1996, 2, 772.

16. Danopoulos, A. A.; Winston, S.; Gelbrich, T.; Hursthouse, M. B.; Tooze, R.P. Chem. Commun. 2002, 482.

17. Caballero, A.; DiezBarra, E.; Jalon, F. A.; Merino, S.; Tejeda, J. J. Organomet. Chem. 2001, 617, 395.

18. Danopoulos, A. A.; Winston, S.; Motherwell, W. B. Chem. Commun. 2002, 1376.

19. Buzinet, P.; Yap, G. P. A.; Richeson, D. S. J. Am. Chem. Soc. 2003, 125, 13314.

20. Hahn, F. E.; Paas, M.; Le, Van D.; Lugger, T. Angew. Chem. Int. Ed. 2003, 42, 5243.

21. Merceron-Saffon, N.; Baceiredo, A.; Gornitzka, H.; Bertrand, G. Science 2003, 301, 1223.

22. For a review, see Herrmann, W. A.; Kocher, K. Angew. Chem. Int. Ed. 1997, 36, 2162.

23. For a review, see Bourissou, D.; Guerret, O.; Gabbaï, F. P.; Bertrand, G. Chem. Rev. 2000, $100,39$.

24. (a) Tomioka H. Advances in Carbene Chemistry (Series: Advances in Carbene Chemistry), 1998, vol. 2, p. 175. (b) Hirai, K.; Tomioka, H. J. Am. Chem. Soc. 1999, 121, 10213. (c) Kirmse W. Angew. Chem. Int. Ed. 2003, 42, 2117.

25. Dias H. V. R., Jin W. Tetrahedron. Lett. 1994, 35, 1365.

26. Korotkikh, N. I.; Rayenko, G. F.; Kiselyov, A. V.; Knishevitsky, A. V.; Shvaika, O. P. Chemistry of Heterocyclic Compounds (Latvia) 2003 (submitted).

27. (a) Korotkikh, N. I.; Rayenko, G. F.; Shvaika, O. P. Rep. Ukr. Acad. Sci. 2000, 2, 135. (b) Korotkikh, N. I.; Rayenko, G. F.; Pekhtereva, T. M.; Shvaika, O. P. Rep. Ukr. Acad. Sci. 1998, 6, 149. (c) Korotkikh, N. I.; Rayenko, G. F.; Shvaika, O. P.; Pekhtereva, T. M.; Cowley, A. H.; Jones, J. N.; MacDonald, C. L. B. J. Org. Chem. 2003, 68, 5762. (d) Korotkikh, N. I.; Rayenko, G. F.; Shvaika, O. P. Report at the 17th Congress on Heterocyclic Chemistry, Vienna, 1999, PO-383. 
28. Rayenko, G. F.; Korotkikh, N. I.; Pekhtereva, T. M.; Shvaika, O. P. J. Org. Chem. (Russia) J. Org. Chem. (Russia) 2000, 37, 1212.

29. Cardin, D. J.; Doyle, M. J.; Lappert, M. F. J. Chem. Soc. Chem. Commun. 1972, 927.

30. Schonherr, H. J.; Wanzlick, H. W., Liebigs Ann. 1970, 103, 1037.

31. (a) Korotkikh, N. I.; Rayenko, G. F.; Shvaika, O. P.; Kiselyov, A. V.; Knishevitsky, A. V.; Cowley A. H.; Macdonald, C. L. B. Rep. at the International Confer. "Reaction Mechanisms and Organic Intermediates", Saint-Petersburg (Russia), June 11-14, 2001, p 73. (b) Korotkikh, N. I.; Rayenko, G. F.; Shvaika, O. P. Rep. at the Russian Confer. of Heterocyclic Chemistry of A.N. Kost Memory, Suzdal (Russia), June 11-14, 2000, p 230. (c) Korotkikh, N. I.; Kiselyov, A. V.; Rayenko, G. F.; Olyinik N. M., Shvaika, O. P. Rep. Ukr. Acad. Sci. 2002, 6, 142.

32. Levin, M. J.; Scorobogatova, M. S. Chemistry of Heterocyclic Compounds (Latvia) 1967, 339.

33. Shvaika, O. P.; Korotkikh, N. I.; Tereshchenko, G. F.; Kovach, N. A. Chemistry of Heterocyclic Compounds (Latvia) 1976, 6, 853.

34. Korotkikh, N. I.; Rayenko, G. F.; Aslanov, A. F.; Shvaika, O. P. Rep. at the 17-th Ukr. Confer. of Org. Chem. Kharkiv, 1995, Vol. 1, p. 86.

35. Arduengo, A. J.; Calabrese, J. C.; Davidson, F.; Dias H. V. R., Goerlich, J. R.; Krafczyk R., Marshall, W. J., Tamm, M.; Schmutzler R. Helv. Chim. Acta 1999, 82, 2348.

36. Alder, R. W.; Allen, P. R.; Williams, S. J. J. Chem. Soc., Chem. Commun. 1995, 12, 1267.

37. Bordwell, F. G.; Satish, A.V. J. Amer. Chem. Soc. 1991, 113, 3, 985.

38. (a) Moss, R. A.; Shen, S.; Wlostowski, M. Tetrahedron Lett. 1988, 29, 6417. (b) Du, X. M.; Fan, H.; Goodman, J. L.; Kesselmayer, M. A.; Krogh-Jespersen, K.; LaVilla, J. A.; Moss, R. A.; Shen, S.; Sheridan, R. S. J. Am. Chem. Soc. 1990, 112, 1920. See also (c) Pezacki, J. P. Can. J. Chem. 1999, 77, 1230.

39. Alcaraz, G.; Wecker, U.; Baceiredo, A.; Dahan, F.; Bertrand, G., Angew. Chem. Int. Ed. 1995, 34, 1246.

40. Kiselyov, A. S.; Strekowsky, L. Tetrahedron Letters 1994, 35, 207.

41. Hahn, F. E.; Wittenbecher, L.; Boese, R.; Blaser, D. Chem. Eur. J. 1999, 82, 2348.

42. Shvaika, O. P.; Korzhenevskaya, L. M.; Litvinenko, L. M.; Popov, A. F.; Snagoshchenko, L. P. Rep. Acad. Sci. USSR 1983, 273, 360.

43. Guerret, O.; Sole, S.; Gornitzka, H.; Teichert, M.; Trinquier, G.; Bertrand, G. J. Amer. Chem. Soc. 1997, 119, 6668.

44. Chen, W.; Liu, F. J. Organomet. Chem. 2003, 673, 5.

45. Melaiye, A.; Simons, R.S.; Milsted, A.; Pingitore, F.; Wesdemiotis, C.; Tessier, C.A.; Yongs, W. J. J. Med. Chem. 2004, 47, 973.

46. (a) Korotkikh, N. I.; Kiselyov, A. V.; Pekhtereva, T. M.; Shvaika, O. P., Cowley, A. H.; Jones J. N. Rep. Ukr. Acad. Sci. 2004 (submitted). (b) Korotkikh, N.I.; Kiselyov, A.V.; Shvaika O.P. Rep. at the International Confer. "Chemistry of Nitrogen containing heterocycles ”, Kharkiv (Ukraine), September 30 - October 3, 2003, 174. 
47. Arduengo, A. J. III '96 ORCHEM (Bad Nauheim, Germany) "Nucleophile Carbene: Gestern, heute und morgen", September, 1996.

48. Boche, G.; Hilf, C.; Harms, K.; Marsch, M.; Lorenz, J. C. W. Angew. Chem. Int. Ed. 1995, 34,4 .

49. Wacker, A.; Pritzkow, H.; Siebert, W. Eur. J. Inorg. Chem. 1998, 843.

50. Arduengo, A. J.; Goerlich, J. R.; Marshall, W. J. J. Amer. Chem. Soc. 1995, 117, 11027.

51. Arduengo, A. J.; Dias, H. V. R.; Dixon, D. A.; Harlow, R. L., Klooster, W. T.; Koetzle, T. F. J. Am. Chem. Soc. 1994, 116, 6812.

52. Abernethy, C. D.; Clyburne, J. A. C.; Cowley, A. H.; Jones, R. A. J. Am. Chem. Soc. 1999, $121,2329$.

53. Abernethy, C. D.; Cowley, A. H.; Jones, R. A. J. Organomet. Chem. 2000, 596, 3.

54. Kim, Y. J.; Streitwieser, A. J. Am. Chem. Soc. 2002, 124, 5757.

55. Unpublished author's data.

56. (a) Hartzler, H. D. J. Am. Chem. Soc. 1970, 92, 1413. (b) Pazdro, K. M.; Polaczkowa, W., Rocz. Chem. 1971, 54, 1487.

57. Fischer, O.; Rigaud, M. Ber. 1901, 34, 4203; ibid.1902, 35, 1258.

58. Bunting, J.V. Advances in Heterocyclic Chemistry. N-Y: Academic Press. 1979; Vol. 25, pp 2-82.

59. (a) Vorsanger, H. Bull. Soc. Chim. France. 1964, 3118; (b) ibid. 1967, 551; (c) ibid. 1967, 556; (d) ibid. 1967, 2124.

60. Smith, C. W.; Rasmussen, R. S.; Ballard, S. A. J. Am. Chem. Soc. 1949, 71,1082.

61. Patchornick, A.; Berger, A.; Katchalski, E. J. Am. Chem. Soc. 1957, 79, 7416.

62. Konstantinchenko, A. A.; Morkovnick, A. S.; Pozharsky, A. F.; Tertov, B. A. Chemistry of Heterocyclic Compounds (Latvia) 1985, 12, 1694.

63. Rayenko, G. F. Candidate of chemistry thesis, IPOCC Ukr. Nat. Acad. Sci., Donetsk, 2000.

64. Arduengo, A. J.; Gamper, S. F.; Tamm, M.; Calabrese, J. C.; Davidson, F.; Craig, H. A. J. Am. Chem. Soc. 1995, 117, 572.

65. Korotkikh, N. I.; Rayenko, G. F.; Shvaika, O. P. Rep. Ukr. Acad. Sci. 2001, 11, 130.

66. (a) Kirmse, W. Chemistry of carbenes, Academic Press, N-Y.-London, 1964. (b) Nefedov, O. M.; Ioffe, A. I., Menchikov L. G. Chemistry of Carbenes, Chemistry: Moscow, 1990.

67. (a) Zupancic, J. J.; Grasse, P. B.; Lapin, S. C.; Schuster, G. B., Tetrahedron 1985, 41, 1471.

(b) Griller, D.; Nazran, A. S.; Scaiano, J. C., Tetrahedron 1985, 41, 1525. (c) Wong, P. C.; Griller, D.; Scaiano, J. C., J. Am. Chem. Soc. 1982, 104, 6631. (d) Griller, D.; Hadel, L.; Nazran, A. S.; Platz, M. S.; Wong, P. C.; Savino, T. G.; Scaiano, J. C. J. Am. Chem. Soc. 1984, 106, 2227.

68. (a) Wanzlick, H. W.; Schickora, E. Chem. Ber. 1961, 94, 2389. (b) Wanzlick, H. W.; Kleiner, H.-J. Chem. Ber. 1963, 96, 3094. (c) Wanzlick, H. W.; Ahrens, H. Chem. Ber. 1964, 97, 2447. (d) Wanzlick, H. W.; Ahrens, H. Chem. Ber. 1966, 99, 1580.

69. Korotkikh, N. I.; Knishevitsky, A. V.; Pekhtereva, T. M.; Shvaika, O. P. Ukr. Chem. J. 2004 (submitted) 
70. For a review, see (a) Fokin, A. V.; Kolomiyets, A. F. Chemistry of thiiranes, Science, Moscow 1978; (b) Korotkikh N. I. Azacyclic derivatives of small rings: synthesis and properties. Doctor of Sciences thesis, Donetsk, 1997.

71. Carmalt, C. J.; Cowley, A. H. Advances in Inorganic Chemistry, (Series: Advances in Inorganic Chemistry) 2000, 50, 1.

72. Shvaika, O. P.; Korotkikh, N. I., Aslanov, G. F. Rep. Ukr. Acad. Sci., Ser. B. 1990, 5, 46.

73. Korotkikh, N. I.; Rayenko, G. F.; Aslanov, G. F.; Shvaika, O. P. Chemistry of Heterocyclic Compounds (Latvia) 1994, 5, 706.

74. Jones, W. M.; Stowe, M. E.; Well, S. E. E.; Lester, E. W. J. Am. Chem. Soc. 1968, 90, 1849.

75. Kuhn, N.; Steimann, M.; Weyers, G. Z. Naturforsch. 1999, 54b, 427.

76. Korotkikh, N. I. Rus. Chem. J. 1999, 1, 138.

77. Aslanov, A. F.; Korotkikh, N. I.; Shvaika, O. P. Rep. Ukr. Acad., Sci. Ser. B. 1992, 4, 92.

78. (a) Denk, M. K.; Hatano, K.; Ma, M. Tetrahedron Lett. 1999, 40, 2057. (b) Liu, Y.; Lindner, P. E., Lemal, D. M. J. Am. Chem. Soc. 1999, 121, 10626. (c) Hahn, F. E.; Wittenbecher, L.; Van, D. L.; Frolich, R. Angew. Chem. Int. Ed. 2000, 39, 541. (d) Bohm, V. P. W.; Herrmann, W. A. Angew. Chem. Int. Ed. 2000, 39, 4036. (e) Liu, Y. F.; Lemal, D. M. Tetrahedron Letters 2000, 41, 599.

79. Arduengo, A. J. III; Dias, H. V. R.; Calabrese, J. C.; Davidson, F. Organometallics 1993, 12, 3405.

80. (a) Teles, J. H. Breuer, K., Enders, D.; Gielen, H. Synth. Commun. 1999, 29, 1. (b) Enders, D.; Breuer, K., Kallfass, U.; Bahlensiefer, T. Synthesis 2003, 8, 1292.

\section{Biographical Sketch}

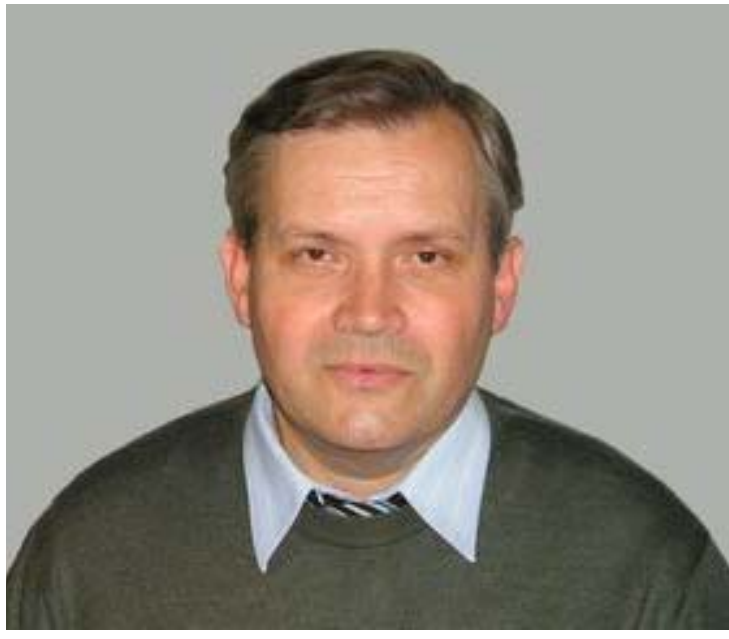

Nikolai Korotkikh was born in Donetsk, Ukraine, in 1951. He was educated at the Donetsk State University, Ukraine (1968-1973), obtained his Candidate of Chemistry (Ph.D.) degree in the Institute of Physical Organic \& Coal Chemistry in 1982, and Doctor of Sciences degree in 1998. After defending a Doctor of Sciences thesis in 1997 he was elected to the position of Head of the Department of Azole Chemistry in 2000 that he holds up to now. Current research is connected with the synthesis, structures, and reactivities of heteroaromatic carbenes, carbenoids and their 
analogues. Earlier works were devoted to the synthesis of heterocyclic derivatives of small rings, ring transformations of heterocycles, materials relevant to the pharmaceutical and polymer industries. He has about 160 publications.

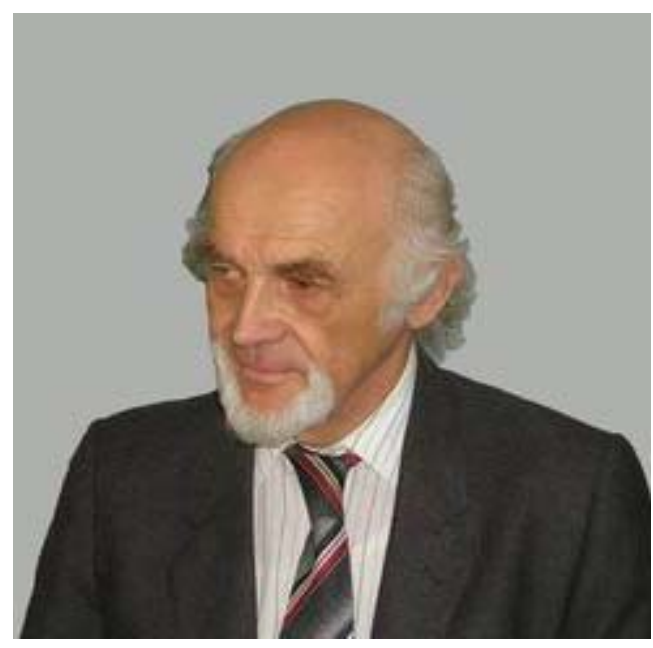

Oles Shvaika was born in Ukraine, in 1932. He completed the Lviv Polythechnical Institute, Ukraine, in 1953, obtained his Candidate of Chemistry (Ph.D.) degree at the Kharkiv State University in 1963, and Doctor of Sciences degree in 1977 at Kiev State University, became Professor in 1988. He had been Head of the Department of Azole Chemistry in the L.M.Litvinenko Institute of Physical Organic and Coal Chemistry in Donetsk for 22 years. His Doctor of Sciences thesis and major scientific works were devoted to ring transformations of heterocyclic compounds especially azoles, azolidines and azolium salts, later to the synthesis of heterocyclic derivatives of small rings. In 90-th years his interest was directed to chemistry of heteroaromatic carbenes. In this part of heterocyclic chemistry, electrochemical research of heteroaromatic salts association in solutions and the applied chemistry concerning pharmaceutical, polymer and optical technique he made about 250 publications.

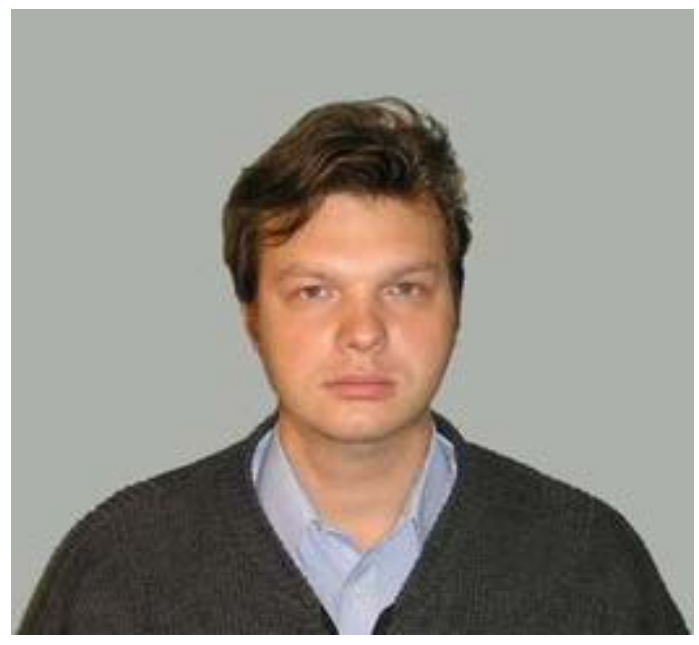

Gennady Rayenko was born in Donetsk, Ukraine, in 1970. He was educated at the Donetsk State University, Ukraine (1987-1992), obtained his Candidate of Chemistry (Ph.D.) degree in the L.M.Litvinenko Institute of Physical Organic \& Coal Chemistry in 2000. In 2001 he was appointed to the position of Scientific Secretary of the Institute that he holds up to now. Current research is concerned with the synthesis, structures, and reactivities of heteroaromatic carbenes and their analogues, chemistry of azolines. He made 35 publications.

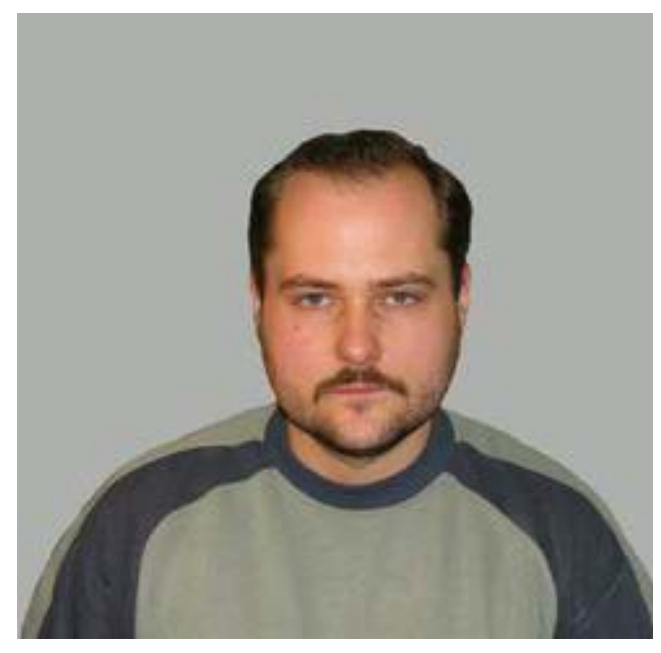

Artyom Kiselyov was born in Artyomovsk, Ukraine, in 1977. He was educated at the Donetsk State University, Ukraine (1994-1999). In 2003 he was elected to the position of Junior Researcher of the L.M.Litvinenko Institute of Physical Organic \& Coal Chemistry. Current research is connected with the synthesis, structures, and 
reactivities of heteroaromatic carbenes and their metal complexes. He has 17 publications.

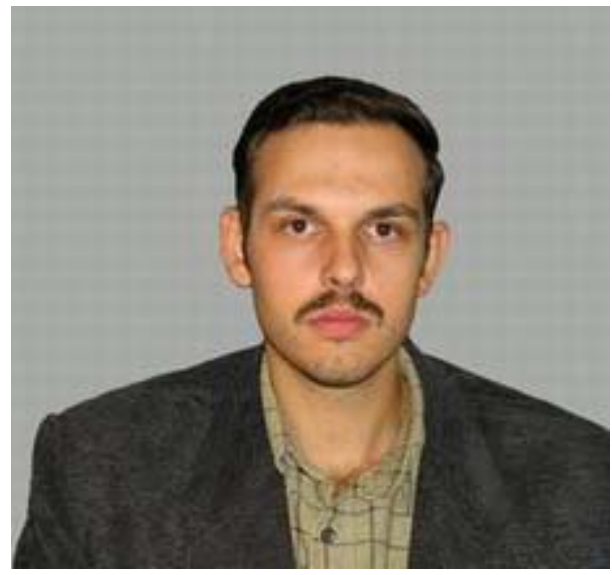

Arthur Knishevitsky was born in Donetsk, Ukraine, in 1978. He was educated at the Donetsk State University, Ukraine 1995-2000 and obtained his B.Sc. and M.Sc. degree in 2001. In 2003 he was elected to the position of Junior Researcher of the L.M.Litvinenko Institute of Physical Organic \& Coal Chemistry. Current research is connected with the synthesis, structures, and reactivities of heteroaromatic carbenes and their analogues. He has 17 publications.

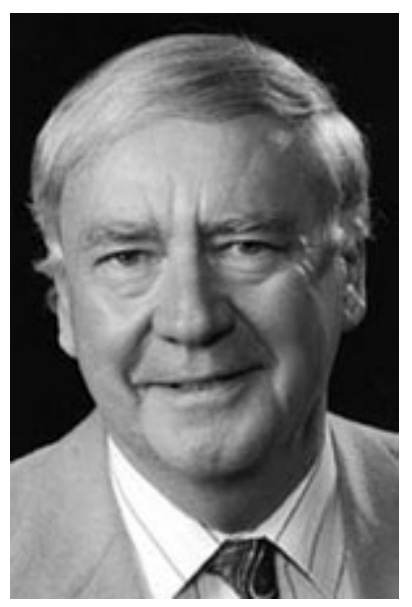

Alan H. Cowley was born in Manchester, U.K. He attended the University of Manchester from 1952 until 1958, where he received B.Sc., M.Sc. and Ph.D. degrees. After a postdoctoral sojourn at the University of Florida, he returned to the U.K. in 1960 where he was employed as a research chemist by Imperial Chemical Industries until 1962. Most of his academic career has been spent at the University of Texas at Austin where he holds the Robert A. Welch Chair in Chemistry. He has close to 500 publications, most of which are concerned with synthetic, structural, and theoretical main group chemistry. He was elected a Fellow of the Royal Society in 1988 and received an Honorary Doctorate from the University of Bordeaux I in 2003. He has served on the Board of Trustees of the Gordon Research Conferences and on several Editorial Boards, including the Journal of the American Chemical Society, Inorganic Chemistry, and Organometallics.

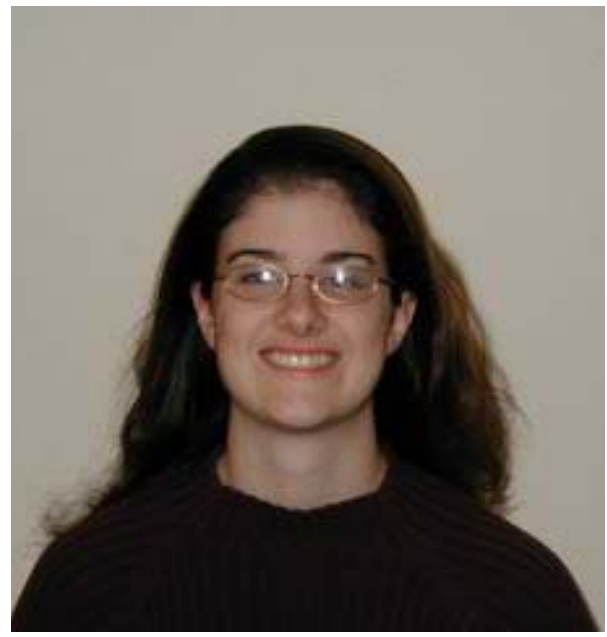

Jamie N. Jones was born in Knoxville, Tennessee. She attended the University of Tennessee from 1996 to 2000, where she received a B.S. in chemistry degree. In August 2004, Jamie received her Ph.D. in chemistry degree from the University of Texas at Austin under the advisement of Professor Alan H. Cowley. During her Ph.D. Jamie was awarded the National Defense Science and Engineering Graduate Fellowship. She currently works for Milliken \& Co. in Spartanburg, South Carolina as a Research Chemist. 


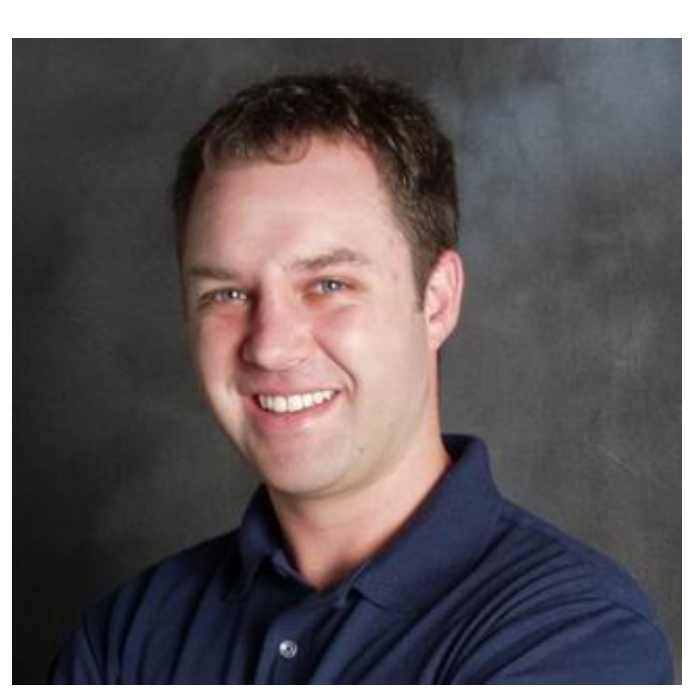

Charles L. B. Macdonald was born in Halifax, Nova Scotia, Canada in 1972. He obtained a B.Sc. in 1994 and a Ph.D. in 1998 from Dalhousie University in Halifax. His doctoral work was conducted in the research group of Neil Burford and his dissertation was awarded the Kenneth T. Leffek Prize. His studies were supported by several scholarships including awards from the Natural Sciences and Engineering Research Council and the Killam Trusts. From 1998 to 2001, he worked as a postdoctoral fellow in Alan H. Cowley's research group at the University of Texas at Austin and he subsequently joined the faculty at the University of Windsor in Windsor, Ontario, Canada. He has more than 40 publications, most of which are concerned with synthetic, structural and theoretical main group chemistry. 